\title{
High-order Semantic Role Labeling
}

\author{
Zuchao Li $^{1,2,3}$, Hai Zhao ${ }^{1,2,3, *}$, Rui Wang ${ }^{4}$, and Kevin Parnow ${ }^{1,2,3}$ \\ ${ }^{1}$ Department of Computer Science and Engineering, Shanghai Jiao Tong University (SJTU) \\ ${ }^{2}$ Key Laboratory of Shanghai Education Commission for Intelligent Interaction \\ and Cognitive Engineering, Shanghai Jiao Tong University, Shanghai, China \\ ${ }^{3}$ MoE Key Lab of Artificial Intelligence, AI Institude, Shanghai Jiao Tong University, China \\ ${ }^{4}$ National Institute of Information and Communications Technology (NICT), Kyoto, Japan \\ charleedsjtu.edu.cn, zhaohai@cs,sjtu.edu.cn, wangruienict.go.jp, parnow@sjtu.edu.cn
}

\begin{abstract}
Semantic role labeling is primarily used to identify predicates, arguments, and their semantic relationships. Due to the limitations of modeling methods and the conditions of pre-identified predicates, previous work has focused on the relationships between predicates and arguments and the correlations between arguments at most, while the correlations between predicates have been neglected for a long time. High-order features and structure learning were very common in modeling such correlations before the neural network era. In this paper, we introduce a highorder graph structure for the neural semantic role labeling model, which enables the model to explicitly consider not only the isolated predicate-argument pairs but also the interaction between the predicate-argument pairs. Experimental results on 7 languages of the CoNLL-2009 benchmark show that the highorder structural learning techniques are beneficial to the strong performing SRL models and further boost our baseline to achieve new stateof-the-art results.
\end{abstract}

\section{Introduction}

Linguistic parsing seeks the syntactic/semantic relationships between language units, such as words or spans (chunks, phrases, etc.). The algorithms usually use factored representations of graphs to accomplish the target: a set of nodes and relational

* Corresponding author. This paper was partially supported by National Key Research and Development Program of China (No. 2017YFB0304100), Key Projects of National Natural Science Foundation of China (U1836222 and 61733011), Huawei-SJTU Long Term AI Project, Cuttingedge Machine Reading Comprehension and Language Model. Rui Wang was partially supported by JSPS grant-in-aid for early-career scientists (19K20354): "Unsupervised Neural Machine Translation in Universal Scenarios" and NICT tenuretrack researcher startup fund "Toward Intelligent Machine Translation". arcs. The types of features that the model can exploit in the inference depend on the information included in the factorized parts.

Before the introduction of deep neural networks, in syntactic parsing (a kind of linguistic parsing), several works (McDonald and Pereira, 2006; Carreras, 2007; Koo and Collins, 2010; Zhang and McDonald, 2012; Ma and Zhao, 2012) showed that high-order parsers utilizing richer factorization information achieve higher accuracy than low-order ones due to the extensive decision history that can lead to significant improvements in inference (Chen et al., 2010).

Semantic role labeling (SRL) (Gildea and Jurafsky, 2002; Zhao and Kit, 2008; Zhao et al., 2009b, 2013) captures the predicate-argument structure of a given sentence, and it is defined as a shallow semantic parsing task, which is also a typical linguistic parsing task. Recent high-performing SRL models (He et al., 2017; Marcheggiani et al., 2017; He et al., 2018a; Strubell et al., 2018; He et al., 2018b; Cai et al., 2018), whether labeling arguments for a single predicate using sequence tagging model at a time or classifying the candidate predicateargument pairs, are (mainly) belong to first-order parsers. High-order information is an overlooked potential performance enhancer; however, it does suffer from an enormous spatial complexity and an expensive time cost in the inference stage. As a result, most of the previous algorithms for highorder syntactic dependency tree parsing are not directly applicable to neural parsing. In addition, the target of model optimization, the high-order relationship, is very sparse. It is not as convenient for training the model with negative likelihood as the first-order structure is because the efficient gradient backpropagation of parsing errors from the high-order parsing target is indispensable in neural parsing models.

To alleviate the computational and graphic mem- 

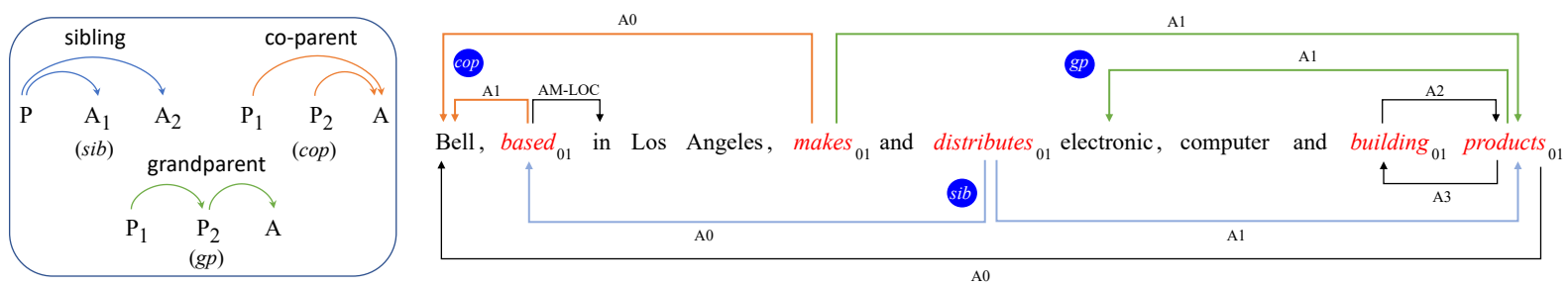

Figure 1: Left is the second-order parts (structures) considered in this paper, where the $P$ stands for a predicate, and $A$ stands for argument. Right is an example of semantic role labeling from the CoNLL-09 training dataset.

ory occupation challenges of explicit high-order modeling in the training and inference phase, we propose a novel high-order scorer and an approximation high-order decoding layer for the SRL parsing model. For the high-order scorer, we adopt a triaffine attention mechanism, which is extended from the biaffine attention (Dozat and Manning, 2017), for scoring the second-order parts. In order to ensure the high-order errors backpropagate in the training stage and to output the part score of the first-order and highest-order fusion in the highest-scoring parse search stage during decoding, inspired by (Lee et al., 2018; Wang et al., 2019), we apply recurrent layers to approximate the highorder decoding iteratively and hence make it differentiable.

We conduct experiments on popular English and multilingual benchmarks. From the evaluation results on both test and out-of-domain sets, we observe a statistically significant increase in semantic$F_{1}$ score with the second-order enhancement and report new state-of-the-art performance in all test set of 7 languages except for the out-of-domain test set in English. Additionally, we also evaluated the results of the setting without pre-identified predicates and compared the effects of every different high-order structure combination on all languages to explore how the high-order structure contributes and how its effect differs from language to language. Our analysis of the experimental results shows that the explicit higher-order structure learning yields steady improvements over our replicated strong BERT baseline for all scenarios.

\section{High-order Structures in SRL}

High-order features or structure learning is known to improve linguistic parser accuracy. In dependency parsing, high-order dependency features encode more complex sub-parts of a dependency tree structure than the features based on first-order, bigram head-modifier relationships. The clear trend in dependency parsing has shown that the addition of such high-order features improves parse accuracy (McDonald and Pereira, 2006; Carreras, 2007; Koo and Collins, 2010; Zhang and McDonald, 2012; Ma and Zhao, 2012). We find that this addition can also benefit semantic parsing, as a tree is a specific form of a graph, and the high-order properties that exist in a tree apply to the graphs in semantic parsing tasks as well.

For a long time, SRL has been formulated as a sequential tagging problem or a candidate pair (word pair) classification problem. In the pattern of sequential tagging, only the arguments of one single predicate are labeled at a time, and a CRF layer is generally considered to model the relationship between the arguments implicitly (Zhou and Xu, 2015). In the candidate pair classification pattern, He et al. (2018a) propose an end-to-end approach for jointly predicting all predicates, arguments, and their relationships. This pattern focuses on the first-order relationship between predicates and arguments and adopts dynamic programming decoding to enforce the arguments' constraints. From the perspective of existing SRL models, high-order information has long been ignored. Although current first-order neural parsers could encode the highorder relationships implicitly under the stacking of the self-attention layers, the advantages of explicit modeling over implicit modeling lie in the lower training cost and better stability. This performance improvement finding resultant of high-order features or structure learning suggests that the same benefits might be observed in SRL. Thus, this paper intends to explore the integration and effect of high-order structures learning in the neural SRL model.

The trade-offs between rich high-order structures (features), decoding time complexity, and memory requirements need to be well considered, especially in the current neural models. The work of $\mathrm{Li}$ et al. (2020) suggests that with the help of deep neural 
network design and training, exact decoding can be replaced with an approximate decoding algorithm, which can significantly reduce the decoding time complexity at a very small performance loss; however, using high-order structure unavoidably brings problematically high graphic memory demand due to the gradient-based learning methods in the neural network model. Given an input sentence with length $L$, order $J$ of parsing model, the memory required is $O\left(L^{J+1}\right)$. In the current GPU memory conditions, second-order $J=2$ is the upper limit that can be explored in practice if without pruning. Therefore, we enumerate all three second-order structures as objects of study in SRL, as shown in the left part of Figure 1, namely sibling (sib), co-parents (cop), and grandparent (gp).

As shown in the SRL example presented in right part of Figure 1, our second-order SRL model looks at several pairs of arcs:

- sibling (Smith and Eisner, 2008; Martins et al., 2009): arguments of the same predicate;

- co-parents (Martins and Almeida, 2014): predicates sharing the same argument;

- grandparent (Carreras, 2007): predicate that is the argument of another predicate.

Though some high-order structures have been studied by some related works (Yoshikawa et al., 2011; Ouchi et al., 2015; Shibata et al., 2016; Ouchi et al., 2017; Matsubayashi and Inui, 2018) in Japanese Predicate Argument Structure (PAS) (Iida et al., 2007) analysis and English SRL (Yang and Zong, 2014), the integration of multiple high-order structures into a single framework and exploring the high-order effects on multiple languages, different high-order structure combinations in a comprehensive way on popular CoNLL-2009 benchmark is the first considered in this paper and thus takes the shape of the main novelties of our work.

\section{Model}

\subsection{Overview}

SRL can be decomposed into four subtasks: predicate identification, predicate disambiguation, argument identification, and argument classification. Since the CoNLL-2009 shared task identified all predicates beforehand, we mainly focus on identifying arguments and labeling them with semantic roles. We formulate the SRL task as a set of arc (and label) assignments between part of the words in the given sentence instead of focusing too much on the roles played by the predicate and argument individually. The predicate-argument structure is regarded as a general dependency relation, with predicate as the head and argument as the dependent (dep) role. Formally, we describe the task with a sequence $X=w_{1}, w_{2}, \ldots, w_{n}$, a set of unlabeled $\operatorname{arcs} Y_{\text {arc }}=\mathcal{W} \times \mathcal{W}$, where $\times$ is the cartesian product, and a set of labeled predicate-argument relations $Y_{\text {label }}=\mathcal{W} \times \mathcal{W} \times \mathcal{R}$ which, along with the set of arcs, is the target to be predicted by the model. $\mathcal{W}=\left\{w_{1}, w_{2}, \ldots, w_{n}\right\}$ refers to the set of all words, and $\mathcal{R}$ is the candidate semantic role labels.

Our proposed model architecture for secondorder SRL is shown in Figure 2, which is inspired and extended from (Lee et al., 2018; Li et al., 2019a; Wang et al., 2019) ${ }^{1}$. The baseline is a first-order SRL model (Li et al., 2019a), which only considers predicate-argument pairs. Our proposed model composes of three modules: contextualized encoder, scorers, and variational inference layers. Given an input sentence, it first computes contextualized word representations using a BiLSTM encoder on the concatenated embedding. The contextualized word representations are then fed into three scorers to give the arc score, arc label score, and high-order part score following the practice of Dozat and Manning (2017). Rather than looking for a model in which exact decoding is tractable, which could be even more stringent for parsing semantic graphs than for dependency trees, we embrace approximate decoding strategies and introduce the variational inference layers to make the high-order error fully differentiable.

\subsection{Encoder}

Our model builds the contextualized representations by using a stacked bidirectional Long Shortterm Memory neural network (BiLSTM) (Hochreiter and Schmidhuber, 1997) to encode the input sentence. Following (He et al., 2018b; Cai et al., 2018; Li et al., 2019a), the input vector is the concatenation of of multiple source embeddings, including a pre-trained word embedding, a random initialized lemma embedding, a predicate indicator embedding, and pre-trained language model layer features; however, unlike their work, we do not use Part-Of-Speech (POS) tag embeddings ${ }^{2}$, which enables our model to be truly syntactic-agnostic.

\footnotetext{
${ }^{1}$ Code available at https://github.com/ bcmi220/hosrl.

${ }^{2} \mathrm{POS}$ tags are also considered to be a kind of syntactic information.
} 


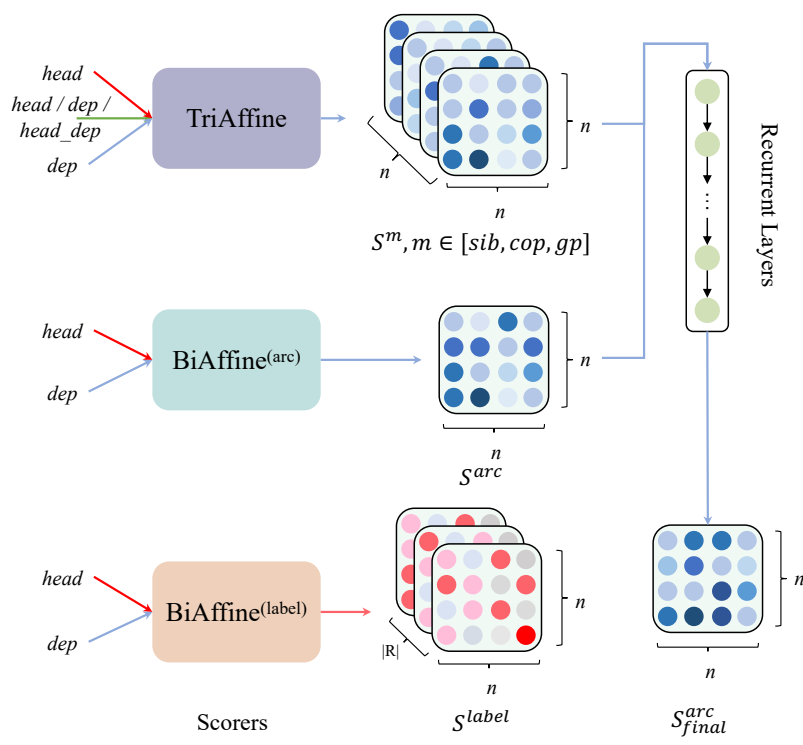

Figure 2: The proposed model architecture.

Additionally, we use pre-trained language model (PLM) layer features because the latest work ( $\mathrm{He}$ et al., 2018b; Li et al., 2018b, 2019a; He et al., 2019) has demonstrated it can boost performance of SRL models. Since these language models were trained at the character- or subword-level, and the out-of-vocabulary (OOV) problem was solved well, we did not use the the bi-directional LSTM-CNN architecture, where convolutional neural networks (CNNs) encode characters inside a word into a character-level representation. Finally, the contextualized representation is obtained as:

$$
H=\operatorname{BiLSTM}(E),
$$

where $e_{i}=e_{i}^{\text {word }} \oplus e_{i}^{\text {lemma }} \oplus e_{i}^{\text {indicator }} \oplus e_{i}^{\text {plm }}$ is the concatenation $(\oplus)$ of the multiple source embeddings of word $w_{i}, E$ represents $\left[e_{1}, e_{2}, \ldots, e_{n}\right]$, and $H=\left[h_{1}, h_{2}, \ldots, h_{n}\right]$ represents the hidden states (i.e., the contextualized representation) of the BiLSTM encoder.

\subsection{Scorers}

Before scoring the arcs and their corresponding role labels, we adopt two multi-layer perceptron (MLP) layers in different scorers to obtain lowerdimensional and role-specific representations of the encoder outputs to strip away irrelevant information from feature extraction.

$$
\begin{aligned}
& h_{i}^{(u-h e a d)}=\mathbf{M L P}^{(u-h e a d)}\left(h_{i}\right), \\
& h_{i}^{(u-d e p)}=\operatorname{MLP}^{(u-d e p)}\left(h_{i}\right), \\
& u \in\{\text { arc }, \text { label }\} .
\end{aligned}
$$

First-order Arc and Label Scorers: In order to score the first-order parts (arcs and labels), we adopt the biaffine classifier proposed by (Dozat and Manning, 2017) to compute the possibility of arc existence and label for dependency $i \rightarrow j$ via biaffine attention.

$$
\begin{aligned}
& \operatorname{BiAF}\left(\mathbf{v}_{i}, \mathbf{v}_{j}\right)=\left[\begin{array}{c}
\mathbf{v}_{j} \\
1
\end{array}\right]^{\mathrm{T}} \mathbf{U}^{l s t} \mathbf{v}_{i} \\
& S_{i, j}^{u}=S_{i \rightarrow j}^{u}=\operatorname{BiAF}^{(u)}\left(h_{i}^{(u-h e a d)}, h_{j}^{(u-d e p)}\right), \\
& u \in\{\text { arc,label }\},
\end{aligned}
$$

where the dimensional size of weight matrix $\mathbf{U}^{1 s t}$ is $(d+1) \times d$ in the $\mathbf{B i A F}{ }^{(a r c)}$ function , and $(d+$ 1) $\times|\mathcal{R}| \times d$ in the $\mathbf{B i A F}{ }^{(\text {label })}$ function, $d$ is the hidden size of the MLPs.

Second-order part scorer: Inspired by (Dozat and Manning, 2017; Wang et al., 2019; Zhang et al., 2020), we extend the original biaffine attention to a triaffine attention for scoring the second-order parts. Similarly, we employ extra MLPs to perform dimension reduction and feature extraction. Additionally, an extra role head_dep apart from head and dep is introduced by the grandparent parts. This role is both the predicate of an argument and the argument of the other predicate.

$$
\begin{aligned}
& h_{i}^{(m-\text { head })}=\mathbf{M L P}^{(m-\text { head })}\left(h_{i}\right), \\
& h_{i}^{(m-d e p)}=\mathbf{M L P}^{(m-d e p)}\left(h_{i}\right), \\
& h_{i}^{(\text {head_dep })}=\mathbf{M L P}^{(\text {head_dep })}\left(h_{i}\right), \\
& m \in\{\text { sib,cop, gp }\} .
\end{aligned}
$$

To reduce the computation and memory cost, we only use an arc triaffine function to compute scores of second-order parts; the label triaffine scorer is not considered. A triaffine function is defined as follows:

$$
\begin{aligned}
& \operatorname{TriAF}\left(\mathbf{v}_{i}, \mathbf{v}_{j}, \mathbf{v}_{k}\right)=\left[\begin{array}{c}
\mathbf{v}_{k} \\
1
\end{array}\right]^{\mathrm{T}} \mathbf{v}_{i}^{\mathrm{T}} \mathbf{U}^{2 n d}\left[\begin{array}{c}
\mathbf{v}_{j} \\
1
\end{array}\right] \\
& S_{i, j, k}^{(s i b)}=S_{i \rightarrow j, i \rightarrow k}^{(s i b)}= \\
& \operatorname{TriAF}^{(s i b)}\left(h_{i}^{(s i b-h e a d)}, h_{j}^{(s i b-d e p)}, h_{k}^{(s i b-d e p)}\right), \\
& S_{i, j, k}^{(c o p)}=S_{i \rightarrow j, k \rightarrow j}^{(c o p)}= \\
& \operatorname{TriAF}^{(c o p)}\left(h_{i}^{(\text {cop-head })}, h_{j}^{(c o p-d e p)}, h_{k}^{(\text {cop-head })}\right), \\
& S_{i, j, k}^{(g p)}=S_{k \rightarrow i \rightarrow j}^{(g p)}= \\
& \quad \operatorname{TriAF}^{(g p)}\left(h_{i}^{(\text {head_dep })}, h_{j}^{(g p-d e p)}, h_{k}^{(g p-h e a d)}\right),
\end{aligned}
$$


where the weight matrix $\mathbf{U}^{2 n d}$ is $(d \times(d+1) \times$ $(d+1))$-dimensional.

\subsection{Variational Inference Layers}

In the first-order model, we adopt the negative likelihood of the golden structure as the loss to train the model, but in the second-order module of our proposed model, a similar approach will encounter the sparsity problem, as the maximum likelihood estimates cannot be obtained when the number of trainable variables is much larger than the number of observations. In other words, it is not feasible to directly approximate the real distribution with the output distribution of the second-order scorer because of the sparsity of the real distribution.

Computing the arc probabilities based on the first-order and multiple second-order scores outputs can be seen as doing posterior inference on a Conditional Random Field (CRF). As exact inference on this CRF is intractable(Wang et al., 2019), we resort to using the variational inference algorithms that allow the model to condition on high-order structures while being fully differentiable.

The variational inference computes the posterior distribution of unobserved variables in the probability graph model. Then, parameter learning is carried out with the observed variables and the predicted unobservable variables. Mean field variational inference approximates a true posterior distribution with a factorized variational distribution and tries to iteratively minimize its KL divergence. Thus, we use mean field variational inference approximates to obtain the final arc distribution. This inference involves $T$ iterations of updating arc probabilities, denoted as $Q_{i, j}^{(t)}$ for the probabilities of arc $i \rightarrow j$ at iteration $t$. The iterative update process is described as follows:

$$
\begin{array}{r}
\mathcal{G}_{i, j}^{(t-1)}=\sum_{k \neq i, j}\left\{Q_{i, k}^{(t-1)} S_{i \rightarrow j, i \rightarrow k}^{(s i b)}+Q_{k, j}^{(t-1)} S_{i \rightarrow j, k \rightarrow j}^{(c o p)}\right. \\
\left.+Q_{k, i}^{(t-1)} S_{k \rightarrow i \rightarrow j}^{(g p)}+Q_{j, k}^{(t-1)} S_{i \rightarrow j \rightarrow k}^{(g p)}\right\},
\end{array}
$$$$
Q_{i, j}^{(t)}= \begin{cases}\exp \left(S_{i \rightarrow j}^{a r c}+\mathcal{G}_{i, j}^{(t-1)}\right), & \text { Arc } i \rightarrow j \text { exist } \\ 1, & \text { Otherwise }\end{cases}
$$

where $\mathcal{G}_{i, j}^{(t-1)}$ is the second-order voting scores, $Q_{i, k}^{(0)}=\operatorname{softmax}\left(S_{i, j}^{a r c}\right)$, and $t$ is the updating step.

Zheng et al. (2015) stated that multiple meanfield update iterations can be implemented by stack-

\begin{tabular}{|c|c|c|c|}
\hline System & Pre-training & WSJ & Brown \\
\hline \multicolumn{4}{|c|}{ w/pre-identified predicate } \\
\hline Cai et al. (2018) & & 89.60 & 79.00 \\
\hline Kasai et al. (2019)* & & 88.60 & 77.60 \\
\hline Zhou et al. (2019) ${ }^{\dagger}$ & & 89.28 & 82.82 \\
\hline He et al. (2019)* & & 89.96 & - \\
\hline Ours & & 90.26 & 80.63 \\
\hline He et al. $(2018 b)^{*}$ & $+\mathrm{E}$ & 89.50 & 79.30 \\
\hline Li et al. (2019a) & $+\mathrm{E}$ & 90.40 & 81.50 \\
\hline Kasai et al. (2019)* & $+\mathrm{E}$ & 90.20 & 80.80 \\
\hline Lyu et al. (2019) & $+\mathrm{E}$ & 90.99 & 82.18 \\
\hline Chen et al. (2019) & $+\mathrm{E}$ & 91.06 & 82.72 \\
\hline Cai and Lapata $(2019)^{\dagger}$ & $+\mathrm{E}$ & 91.20 & 82.50 \\
\hline Ours & $+\mathrm{E}$ & 91.44 & 83.28 \\
\hline Zhou et al. (2019) ${ }^{\dagger}$ & $+\mathrm{B}$ & 91.20 & 85.87 \\
\hline Ours & $+\mathrm{B}$ & 91.77 & 85.13 \\
\hline \multicolumn{4}{|c|}{ w/o pre-identified predicate } \\
\hline Cai et al. (2018) & & 85.00 & 72.50 \\
\hline Li et al. (2019a) & & 85.10 & - \\
\hline Zhou et al. $(2019)^{\dagger}$ & & 85.86 & 77.47 \\
\hline Ours & & 86.16 & 74.20 \\
\hline He et al. $(2018 b)^{*}$ & $+\mathrm{E}$ & 83.30 & - \\
\hline Li et al. (2019a) & $+\mathrm{E}$ & 85.30 & 74.20 \\
\hline Ours & $+\mathrm{E}$ & 87.12 & 76.65 \\
\hline Zhou et al. (2019) ${ }^{\dagger}$ & $+\mathrm{B}$ & 88.17 & 81.58 \\
\hline Ours & $+\mathrm{B}$ & 88.70 & 80.29 \\
\hline
\end{tabular}
ing Recurrent Neural Network (RNN) layers, as
Table 1: Semantic- $F_{1}$ score on CoNLL-2009 English treebanks. WSJ is used for evaluating the in-domain performance and Brown for the out-of-domain. "** denotes that the model uses syntactic information for enhancement, and " $\dagger$ " represents the model is trained with other tasks jointly. "+E" stands for using ELMo as pretrained PLM features, “+B” for using BERT.

each iteration takes $Q$ value estimates from the previous iteration and the unary values (first-order scores) in their original form. In this RNN structure, CRF-RNN, the model parameters therefore can be optimized from the second-order error using the standard backpropagation through time algorithm(Rumelhart et al., 1985; Mozer, 1995). Notably, the number of stacked layers is equal to the iteration steps $T$. Since when $T>5$, increasing the number of iterations usually does not significantly improve results (Krähenbühl and Koltun, 2011), training does not suffer from the vanishing and exploding gradient problem inherent to deep RNNs, and this allows us to use a plain RNN architecture instead of more sophisticated architectures such as LSTMs.

\subsection{Training Objective}

The full model is trained to learn the conditional distribution $P_{\theta}(\hat{Y} \mid X)$ of predicted graph $\hat{Y}$ with gold parse graph $Y^{*}$. Since the parse graph can 


\begin{tabular}{lcccccccc}
\hline System & CA & CS & DE & EN & ES & JA & ZH & Avg. \\
\hline w/ pre-identified predicate & & & & & & & & \\
CoNLL-2009 ST & 80.3 & 85.4 & 79.7 & 85.6 & 80.5 & 78.2 & 78.6 & 81.19 \\
Zhao et al. (2009a) & 80.3 & 85.2 & 76.0 & 86.2 & 80.5 & 78.2 & 77.7 & 80.59 \\
Roth and Lapata (2016) & - & - & 80.1 & 87.7 & 80.2 & - & 79.4 & - \\
Marcheggiani et al. (2017) & - & 86.0 & - & 87.7 & 80.3 & - & 81.2 & - \\
Mulcaire et al. (2018) & 79.45 & 85.14 & 69.97 & 87.24 & 77.32 & 76.00 & 81.89 & 79.57 \\
Kasai et al. (2019) & - & - & - & 90.2 & 83.0 & - & - & - \\
Lyu et al. (2019) & 80.91 & 87.62 & 75.87 & 90.99 & 80.53 & 82.54 & 83.31 & 83.11 \\
Cai and Lapata (2019) & - & - & 83.80 & 91.20 & 82.90 & - & 85.00 & - \\
He et al. (2019) & 84.35 & 88.76 & 78.54 & 89.96 & 83.70 & 83.12 & 84.55 & 84.71 \\
He et al. (2019) & 85.14 & 89.66 & 80.87 & 90.86 & 84.60 & 83.76 & 86.42 & 85.90 \\
\hline Our baseline & 84.96 & 90.18 & 76.02 & 89.61 & 83.77 & 82.65 & 85.73 & 84.70 \\
$\quad+\mathrm{HO}^{+\mathrm{B}}$ & 85.37 & 90.60 & 76.41 & 90.26 & 84.39 & 83.25 & 86.02 & 85.19 \\
Our baseline & 86.40 & 91.48 & 85.21 & 91.23 & 86.60 & 85.55 & 88.24 & 87.82 \\
$\quad+\mathrm{HO}^{+\mathrm{B}}$ & $\mathbf{8 6 . 9 0}$ & $\mathbf{9 1 . 9 3}$ & $\mathbf{8 5 . 5 4}$ & $\mathbf{9 1 . 7 7}$ & $\mathbf{8 6 . 9 6}$ & $\mathbf{8 5 . 9 0}$ & $\mathbf{8 8 . 6 9}$ & $\mathbf{8 8 . 2 4}$ \\
\hline w/o pre-identified predicate $^{+\mathrm{B}}$ & & & & & & & & \\
Our baseline & 83.69 & 89.22 & 60.06 & 85.71 & 82.54 & 73.68 & 81.46 & 79.48 \\
$\quad+$ HO $^{+\mathrm{B}}$ & 84.07 & 89.45 & 60.48 & 86.16 & 83.11 & 74.20 & 82.01 & 79.93 \\
Our baseline $^{+\mathrm{B}}$ & 85.12 & 90.72 & 66.70 & 88.05 & 85.50 & 77.94 & 85.38 & 82.77 \\
$\quad+$ HO $^{+\mathrm{B}}$ & $\mathbf{8 5 . 8 2}$ & $\mathbf{9 1 . 2 2}$ & $\mathbf{6 7 . 1 5}$ & $\mathbf{8 8 . 7 0}$ & $\mathbf{8 6 . 0 0}$ & $\mathbf{7 8 . 8 8}$ & $\mathbf{8 5 . 6 8}$ & $\mathbf{8 3 . 3 5}$ \\
\hline
\end{tabular}

Table 2: Semantic-F $F_{1}$ score on the CoNLL-2009 in-domain test set. The first row is the best result of the CoNLL2009 shared task (Hajič et al., 2009). "WE" indicates the model leverages pre-trained ELMo features (only for English), " $+\mathrm{B}$ " indicates the model leverages BERT for all languages.

be factorized to arcs and corresponding labels, the conditional distribution $P_{\theta}(\hat{Y} \mid X)$ is also factorized to $P_{\theta}\left(\hat{Y}^{(a r c)} \mid X\right)$ and $P_{\theta}\left(\hat{Y}^{(\text {label })} \mid X\right)$, given by:

$$
\begin{gathered}
P_{\theta}\left(\hat{Y}^{(\text {arc })} \mid X\right)=\prod_{1 \leq i \leq n, 1 \leq j \leq n} \operatorname{softmax}\left(Q_{i, j}^{(T)}\right), \\
P_{\theta}\left(\hat{Y}^{(\text {label })} \mid X\right)=\prod_{1 \leq i \leq n, 1 \leq j \leq n} \operatorname{softmax}\left(S_{i, j}^{(\text {label })}\right) .
\end{gathered}
$$

where $\theta$ represents the model parameters. The losses to optimize the model are implemented as cross-entropy loss using negative likelihood to the golden parse:

$$
\begin{aligned}
& \mathcal{L}^{(\text {arc })}(\theta)=-\sum_{1 \leq i \leq n, 1 \leq j \leq n} \log P\left(Y_{i, j}^{*(\operatorname{arc})} \mid X\right), \\
& \left.\mathcal{L}^{(\text {label })}(\theta)=-\sum_{(i, j, r) \in Y^{*}} \log P(\langle i \rightarrow j, r\rangle \mid X)\right),
\end{aligned}
$$

where $r \in \mathcal{R}$ is the semantic role label of arc (predicate-argument) $i \rightarrow j$. The final loss is the weighted average of the arc loss $\mathcal{L}^{(\operatorname{arc})}(\theta)$ and the label loss $\mathcal{L}^{(\text {label) }}(\theta)$ :

$$
\mathcal{L}^{(\text {final })}(\theta)=\lambda \mathcal{L}^{(\text {arc })}(\theta)+(1-\lambda) \mathcal{L}^{(\text {label })}(\theta),
$$

where $\lambda$ is the balance hyper-parameter.

\section{Experiments}

\subsection{Setup}

We conduct experiments and evaluate our model on the CoNLL-2009 (Hajič et al., 2009) benchmark datasets including 7 languages: Catalan (CA), Czech (CS), German (DE), English (EN), Spanish (ES), Japanese (JA), and Chinese (ZH). To better compare with previous works, and to bring the model closer to a real-world usage scenario, we consider two SRL setups on all 7 languages: $w /$ pre-identified predicate and w/o pre-identified predicate. In order to compare with most previous models, the former setup follows official requirements and has predicates identified beforehand in the corpora. The latter one is consistent with a real scenario; where the model is required to predict all the predicates and their arguments and is therefore relatively more difficult. Since the predicates need to be predicted in the w/o preidentified predicate setup, we treat the identification and disambiguation of predicates as one sequence tagging task, and we adopt BiLSTM+MLP and BERT+MLP sequence tagging architectures to adapt to different requirements. We directly adopt most hyper-parameter settings and training 


\begin{tabular}{|c|c|c|c|}
\hline System & $\mathrm{P}$ & $\mathrm{R}$ & $F_{1}$ \\
\hline \multicolumn{4}{|c|}{ German } \\
\hline Zhao et al. (2009a) & - & - & 67.78 \\
\hline Lyu et al. (2019) & - & - & 65.69 \\
\hline Our baseline & 71.34 & 67.73 & 69.49 \\
\hline$+\mathrm{HO}$ & 71.66 & 69.36 & 70.49 \\
\hline Our baseline $^{+B}$ & 71.77 & 70.02 & 70.88 \\
\hline$+\mathrm{HO}^{+\mathrm{B}}$ & 72.14 & 71.86 & 72.00 \\
\hline \multicolumn{4}{|c|}{ Czech } \\
\hline Zhao et al. (2009a) & - & - & 82.66 \\
\hline Marcheggiani et al. (2017) & 88.00 & 86.50 & 87.20 \\
\hline Lyu et al. (2019) & - & - & 86.04 \\
\hline Our baseline & 91.22 & 89.88 & 90.54 \\
\hline$+\mathrm{HO}$ & 91.50 & 90.03 & 90.75 \\
\hline Our baseline $\mathrm{e}^{+\mathrm{B}}$ & 91.98 & 91.23 & 91.60 \\
\hline$+\mathrm{HO}^{+\mathrm{B}}$ & 91.87 & 91.61 & 91.74 \\
\hline
\end{tabular}

Table 3: Precision, Recall, and Semantic- $F 1$ scores on German and Czech out-of-domain test sets.

strategy from (Dozat and Manning, 2017; Wang et al., 2019). Please refer to Appendix A.1 for details.

\subsection{Results And Analysis}

Main Results ${ }^{3}$ Table 1 presents the results on the standard English test set, WSJ (in-domain) and Brown (out-of-domain). For a fair comparison with previous works, we report three cases: not using pre-training, using ELMo (Peters et al., 2018), and using BERT (Devlin et al., 2019). Our single model achieves the best performance on the in-domain test set without syntactic information and extra resources for both types of setup, w/ and w/o preidentified predicate. On the out-of-domain test set, even though Zhou et al. (2019) obtains the highest score, their model is joint and likely achieves domain adaptation due to external tasks and resources. In general, our model achieves significant performance improvements in both in-domain and out-of-domain settings, especially while using pretraining out-of-domain. Furthermore, the results of using ELMo and BERT show that the stronger pre-training model brings greater improvement.

Multilingual Results Table 2 summarizes the results on CoNLL-2009 standard in-domain test sets of all 7 languages. The bold results in Table 2 are obtained by averaging the every results from 5 training rounds with different random seeds to avoid random initialization impact on the model. We compare our baseline and full model with previous multilingual works. The performance of our baseline

\footnotetext{
${ }^{3}$ Due to the limited space, we only analyzed the main results. Please refer to Appendix A.2 for detailed results.
}

is similar to the model of He et al. (2019), which integrated syntactic information and achieved the best results. This shows that our baseline is a very strong SRL model, and owes its success to directly modeling on the full semantic graph rather than separately based on predicates. Moreover, our model with the proposed high-order structure learning (+ $\mathrm{HO}$ ) obtains absolute improvements of $0.49 \%$ and $0.42 \% \mathrm{~F} 1$ without pre-training and with BERT, respectively, achieving the new best results on all benchmarks. Because the quantities of high-order structures are different among different languages, consistent improvement on 7 languages already shows that our empirical results are convincing.

In addition, we also report the results of the w/o pre-identified predicate setup for all languages, which is a more realistic scenario. The overall decline without pre-identified predicates shows that predicate recognition has a great impact. Especially for German, the obvious drop is probably because the ratio of predicates in the German evaluation set is relatively small and is sensitive to the model parameters; however, in this setup, our high-order structure learning leads to consistent improvements in all languages with the $w /$ pre-identified predicate setup, demonstrating the effectiveness of the proposed method.

To show the statistical significance of our results, in addition to adopting the above-mentioned common practice in SRL at model-level that reports the average results with multiple runs and random seeds, we further follow the practice in machine translation (Koehn, 2004) to conduct a significant test at example level. We sampled the prediction results for 500 times, 50 sentences each time, and evaluated the sampled subset. The result of $+\mathrm{HO}$ is significantly higher than that of the baseline model $(p<0.01)$, verifying the significance of the results.

Out-of-domain Results Besides English, there are also out-of-domain test sets for German and Czech. To verify the generalization capability of our model, we further conduct experiments on these test sets under $w /$ pre-identified predicates and compare results with existing work (in Table 3). Our model achieves new state-of-the-art results of 70.49\% (German) and $90.75 \%$ (Czech) F1-score, significantly outperforming the previous best system (Lyu et al., 2019). Furthermore, there is even a gain with using pre-trained BERT, showing that BERT can improve the generalization ability of the model. In addition, we observe that the model 


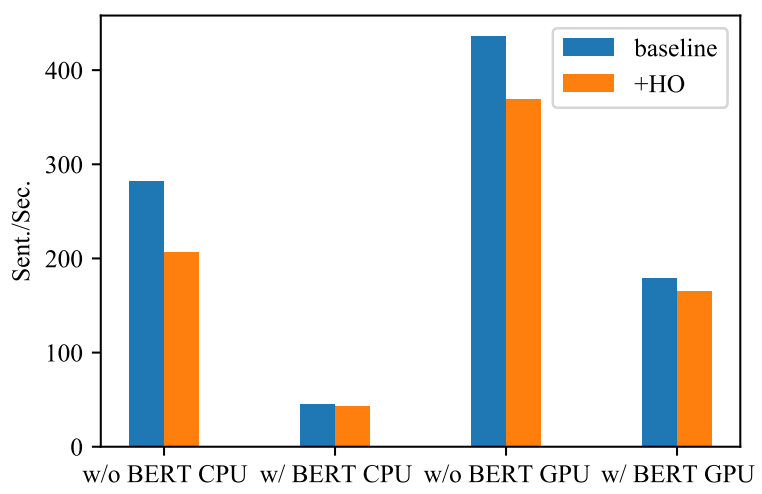

Figure 3: Parsing speed measured on CoNLL-2009 English test set.

$(+\mathrm{HO})$ yields stable performance improvement in recall, which shows the proposed high-order structure learning is beneficial to identifying arguments.

Time Complexity and Parsing Speed The time complexity and parsing speed of high-order models have always been concerns. In our proposed highorder model, the time complexity comes from two parts: one is the matrix operations in the biaffine and triaffine attention $\left(O\left(d_{\mathrm{BiAF}}^{2}\right)\right.$ and $O\left(d_{\mathrm{TriAF}}^{3}\right)$, respectively), where $d_{\mathrm{BiAF}}$ and $d_{\mathrm{TriAF}}$ is the hidden size of the scorer, and the other is the inference procedure $\left(O\left(n^{3}\right)\right)$, making the total time complexity $O\left(d_{\mathrm{BiAF}}^{2}+d_{\mathrm{TriAF}}^{3}+n^{3}\right)$, while, for for our baseline, the full time complexity is $O\left(d_{\mathrm{BiAF}}^{2}+n^{2}\right)$. Additionally, in the case of leveraging pre-trained PLM features, the time complexity of encoders such as BERT is a part that cannot be ignored. We measured the parsing speed of our baseline and high-order models on the English test set, both with BERT pre-training and without, on the CPU and GPU, respectively, with an Intel Xeon 6150 CPU and a Titan V100 GPU. The comparison is shown in Figure 3. Results show that the speed loss of $+\mathrm{HO}$ is $26.7 \%, 5.5 \%, 15.4 \%$ and $7.6 \%$ in the respective four scenarios, while the speed loss brought by BERT is $84.1 \%, 79.5 \%$ on CPU and $60.0 \%$ and $55.2 \%$ on GPU. Therefore, $+\mathrm{HO}$ brings a loss of speed, but with GPU acceleration, the loss ratio is reduced. In the case of BERT pre-training, $+\mathrm{HO}$ is no longer the bottleneck of parsing speed.

High-order Structures Contribution To explore the contribution of high-order structures in depth, we consider all possible combinations of structures and conduct experiments on the English test set under the $w /$ pre-identified predicate setup. Table 4 shows the results of two baseline models

\begin{tabular}{lcclcc}
\hline \multirow{2}{*}{ System } & \multicolumn{2}{c}{ w/o BERT } & & \multicolumn{2}{c}{ w/ BERT } \\
\cline { 2 - 3 } \cline { 5 - 6 } & $\mathrm{P} / \mathrm{R}$ & $\mathrm{F}_{1}$ & & $\mathrm{P} / \mathrm{R}$ & $\mathrm{F}_{1}$ \\
\hline baseline & $91.29 / 88.00$ & 89.61 & & $92.31 / 90.18$ & 91.23 \\
+ sib & $91.46 / 88.53$ & 89.97 & & $92.49 / 90.58$ & 91.53 \\
+cop & $91.40 / 88.45$ & 89.90 & & $92.21 / 90.50$ & 91.35 \\
+ gp & $91.41 / 88.14$ & 89.75 & & $92.77 / 90.09$ & 91.41 \\
+sib+cop & $91.33 / 88.74$ & 90.02 & & $92.36 / 90.84$ & 91.60 \\
+sib+gp & $91.21 / 88.58$ & 89.87 & & $92.64 / 90.44$ & 91.53 \\
+cop+gp & $91.26 / 88.68$ & 89.95 & & $92.37 / 90.35$ & 91.35 \\
+ALL & $91.60 / 88.95$ & 90.26 & & $92.59 / 90.98$ & 91.77 \\
\hline
\end{tabular}

Table 4: Effect of different second-order structures and their combination on model performance.

(with and without BERT pre-training). Using these structures separately improves our model, as shown in $+s i b$ with a $0.36 F_{1}$ gain; however, the further improvement of applying two structures is limited. For example, model $(+s i b)$ performs even better than $(+s i b+g p)$. The reason might be that the $s i b$ (between the arguments) and the $g p$ (between the predicates) are two irrelevant structures. Regardless, we can observe that +ALL (the combination of all three structures) model achieves the best performance ( up to $0.65 F_{1}$ ). One possible reason for the result is that the cop (between arguments and predicates) sets up a bridge for $s i b$ and $g p$ structures. In other words, these observations suggest that the three structure learning may be complementary.

We further explored the sources of the higher order structure's improvement in SRL performance. we split the test set into two parts, one with the high-order relationship (cop and gp), and the other without. Taking the CoNLL09 English test set as an example, the total size of the test set is 2399 sentences, and there are 1936 sentences with highorder relationships. We recalculated Sem-F1 for these two subsets, and found that the scores on the subsets with higher-order relationships are significantly higher than those without( $(0.4 \%$ F-score). It shows that our model does improve the prediction of high-order structure, rather than a specific type of semantic role. For simple sentences (without HO), the baseline can already parse it very well, which also explains the reason why the improvement in some languages is not great.

\section{Related Work}

The CoNLL-2009 shared task advocated performing SRL for multiple languages to promote multilingual NLP applications. (Zhao et al., 2009a) proposed an integrated approach by exploiting large- 
scale feature sets, while (Björkelund et al., 2009) used a generic feature selection procedure, which yielded significant gains in the multilingual SRL shared task. With the development of deep neural networks (Li et al., 2018a; Xiao et al., 2019; Zhou and Zhao, 2019; Zhang et al., 2019c,a; Li et al., 2019c; Luo et al., 2020; Li et al., 2019b; Zhang et al., 2019b) for NLP, most subsequent SRL works have focused on improving the performance of English, with occasional comparisons to other languages (Lei et al., 2015; Swayamdipta et al., 2016; Roth and Lapata, 2016; Marcheggiani et al., 2017; He et al., 2018b; Li et al., 2018b; Cai et al., 2018). Mulcaire et al. (2018) built a polyglot semantic role labeling system by combining resources from all languages in the CoNLL2009 shared task for exploiting the similarities between semantic structures across languages. This approach, while convenient, is still far less effective than separate model training on different languages. Lyu et al. (2019) modeled interactions between argument labeling decisions with a structure refinement network, resulting in an effective model, and outperforming strong factorized baseline models on all 7 languages. He et al. (2019) boosted multilingual SRL performance with special focus on the impact of syntax and contextualized word representations and achieved new state-ofthe-art results on the CoNLL-2009 benchmarks of all languages, resulting in an effective model and outperforming strong factorized baseline models on all 7 languages

High-order parsing is one of the research hotspots in which first-order parsers meet performance bottlenecks; this has been extensively studied in the literature of syntactic dependency parsing(McDonald et al., 2005; McDonald and Pereira, 2006; Carreras, 2007; Koo and Collins, 2010; Martins et al., 2011; Ma and Zhao, 2012; Gormley et al., 2015; Zhang et al., 2020). In semantic parsing, Martins and Almeida (2014) proposed a way to encode high-order parts with hand-crafted features and introduced a novel co-parent part for semantic dependency parsing. Cao et al. (2017) proposed a quasi-second-order semantic dependency parser with dynamic programming. Wang et al. (2019) trained a second-order parser in an end-toend manner with the help of mean field variational inference and loopy belief propagation approximation. In SRL or related research field, there is also some related work on the improvement of performance by high-order structural information. On the Japanese NAIST Predict-Argument Structure (PAS) dataset, some works (Yoshikawa et al., 2011; Ouchi et al., 2015; Iida et al., 2015; Shibata et al., 2016; Ouchi et al., 2017; Matsubayashi and Inui, 2018) mainly studied the relationship between multiple predicates separately, that is, the $g p$ and $c p$ high-order relationship mentioned in our paper. (Yang and Zong, 2014) considered the interactions between predicate-argument pairs on Chinese Propbank dataset. Although the motivation is consistent with our work, we first consider multiple high-order relationships at the same time within a more uniform framework on more popular benchmarks and for more languages.

\section{Conclusion and Future Work}

In this work, we propose high-order structure learning for dependency semantic role labeling. The proposed framework explicitly models high-order graph structures on a strong first-order baseline model while scoring the correlation of predicted predicate-argument pairs. The resulting model achieves state-of-the-art results on all 7 languages in the CoNLL-2009 test data sets except the outof-domain benchmark in English. In addition, we consider both given and not-given predicates on all languages, explore the impact of every high-order structure combinations on performance for all languages, and reveal the adaptive range of high-order structure learning on different languages. In future work, we will continue to explore higher-order structures and pruning strategies to reduce the time complexity and memory occupation.

\section{References}

Mikhail Arkhipov, Maria Trofimova, Yuri Kuratov, and Alexey Sorokin. 2019. Tuning multilingual transformers for language-specific named entity recognition. In Proceedings of the 7th Workshop on BaltoSlavic Natural Language Processing, pages 89-93, Florence, Italy. Association for Computational Linguistics.

Anders Björkelund, Love Hafdell, and Pierre Nugues. 2009. Multilingual semantic role labeling. In Proceedings of the Thirteenth Conference on Computational Natural Language Learning (CoNLL 2009): Shared Task, pages 43-48, Boulder, Colorado. Association for Computational Linguistics.

Piotr Bojanowski, Edouard Grave, Armand Joulin, and Tomas Mikolov. 2017. Enriching word vectors with 
subword information. Transactions of the Association for Computational Linguistics, 5:135-146.

Jiaxun Cai, Shexia He, Zuchao Li, and Hai Zhao. 2018. A full end-to-end semantic role labeler, syntacticagnostic over syntactic-aware? In Proceedings of the 27th International Conference on Computational Linguistics, pages 2753-2765, Santa Fe, New Mexico, USA. Association for Computational Linguistics.

Rui Cai and Mirella Lapata. 2019. Semi-supervised semantic role labeling with cross-view training. In Proceedings of the 2019 Conference on Empirical Methods in Natural Language Processing and the 9th International Joint Conference on Natural Language Processing (EMNLP-IJCNLP), pages 10181027, Hong Kong, China. Association for Computational Linguistics.

Junjie Cao, Sheng Huang, Weiwei Sun, and Xiaojun Wan. 2017. Quasi-second-order parsing for 1endpoint-crossing, pagenumber- 2 graphs. In Proceedings of the 2017 Conference on Empirical Methods in Natural Language Processing, pages 24-34, Copenhagen, Denmark. Association for Computational Linguistics.

Xavier Carreras. 2007. Experiments with a higherorder projective dependency parser. In Proceedings of the 2007 Joint Conference on Empirical Methods in Natural Language Processing and Computational Natural Language Learning (EMNLPCoNLL), pages 957-961, Prague, Czech Republic. Association for Computational Linguistics.

José Cañete, Gabriel Chaperon, Rodrigo Fuentes, and Jorge Pérez. 2020. Spanish pre-trained bert model and evaluation data. In to appear in PMLADC at ICLR 2020.

Wenliang Chen, Jun'ichi Kazama, Yoshimasa Tsuruoka, and Kentaro Torisawa. 2010. Improving graph-based dependency parsing with decision history. In Coling 2010: Posters, pages 126-134, Beijing, China. Coling 2010 Organizing Committee.

Xinchi Chen, Chunchuan Lyu, and Ivan Titov. 2019. Capturing argument interaction in semantic role labeling with capsule networks. In Proceedings of the 2019 Conference on Empirical Methods in Natural Language Processing and the 9th International Joint Conference on Natural Language Processing (EMNLP-IJCNLP), pages 5415-5425, Hong Kong, China. Association for Computational Linguistics.

Jacob Devlin, Ming-Wei Chang, Kenton Lee, and Kristina Toutanova. 2019. BERT: Pre-training of deep bidirectional transformers for language understanding. In Proceedings of the 2019 Conference of the North American Chapter of the Association for Computational Linguistics: Human Language Technologies, Volume 1 (Long and Short Papers), pages 4171-4186, Minneapolis, Minnesota. Association for Computational Linguistics.
Timothy Dozat and Christopher D Manning. 2017. Deep biaffine attention for neural dependency parsing. In International Conference on Learning Representations.

Daniel Gildea and Daniel Jurafsky. 2002. Automatic labeling of semantic roles. Computational linguistics, 28(3):245-288.

Matthew R. Gormley, Mark Dredze, and Jason Eisner. 2015. Approximation-aware dependency parsing by belief propagation. Transactions of the Association for Computational Linguistics, 3:489-501.

Edouard Grave, Piotr Bojanowski, Prakhar Gupta, Armand Joulin, and Tomas Mikolov. 2018. Learning word vectors for 157 languages. In Proceedings of the Eleventh International Conference on Language Resources and Evaluation (LREC 2018), Miyazaki, Japan. European Language Resources Association (ELRA).

Jan Hajič, Massimiliano Ciaramita, Richard Johansson, Daisuke Kawahara, Maria Antònia Martí, Lluís Màrquez, Adam Meyers, Joakim Nivre, Sebastian Padó, Jan Štěpánek, Pavel Straňák, Mihai Surdeanu, Nianwen Xue, and Yi Zhang. 2009. The CoNLL2009 shared task: Syntactic and semantic dependencies in multiple languages. In Proceedings of the Thirteenth Conference on Computational Natural Language Learning (CoNLL 2009): Shared Task, pages 1-18, Boulder, Colorado. Association for Computational Linguistics.

Luheng He, Kenton Lee, Omer Levy, and Luke Zettlemoyer. 2018a. Jointly predicting predicates and arguments in neural semantic role labeling. In Proceedings of the 56th Annual Meeting of the Association for Computational Linguistics (Volume 2: Short Papers), pages 364-369, Melbourne, Australia. Association for Computational Linguistics.

Luheng He, Kenton Lee, Mike Lewis, and Luke Zettlemoyer. 2017. Deep semantic role labeling: What works and what's next. In Proceedings of the 55th Annual Meeting of the Association for Computational Linguistics (Volume 1: Long Papers), pages 473-483, Vancouver, Canada. Association for Computational Linguistics.

Shexia He, Zuchao Li, and Hai Zhao. 2019. Syntaxaware multilingual semantic role labeling. In Proceedings of the 2019 Conference on Empirical Methods in Natural Language Processing and the 9th International Joint Conference on Natural Language Processing (EMNLP-IJCNLP), pages 5350-5359, Hong Kong, China. Association for Computational Linguistics.

Shexia He, Zuchao Li, Hai Zhao, and Hongxiao Bai. 2018b. Syntax for semantic role labeling, to be, or not to be. In Proceedings of the 56th Annual Meeting of the Association for Computational Linguistics (Volume 1: Long Papers), pages 2061-2071, Melbourne, Australia. Association for Computational Linguistics. 
Sepp Hochreiter and Jürgen Schmidhuber. 1997. Long short-term memory. Neural computation, 9(8):1735-1780.

Ryu Iida, Mamoru Komachi, Kentaro Inui, and Yuji Matsumoto. 2007. Annotating a Japanese text corpus with predicate-argument and coreference relations. In Proceedings of the Linguistic Annotation Workshop, pages 132-139, Prague, Czech Republic. Association for Computational Linguistics.

Ryu Iida, Kentaro Torisawa, Chikara Hashimoto, JongHoon Oh, and Julien Kloetzer. 2015. Intrasentential zero anaphora resolution using subject sharing recognition. In Proceedings of the 2015 Conference on Empirical Methods in Natural Language Processing, pages 2179-2189, Lisbon, Portugal. Association for Computational Linguistics.

Jungo Kasai, Dan Friedman, Robert Frank, Dragomir Radev, and Owen Rambow. 2019. Syntax-aware neural semantic role labeling with supertags. In Proceedings of the 2019 Conference of the North American Chapter of the Association for Computational Linguistics: Human Language Technologies, Volume 1 (Long and Short Papers), pages 701-709, Minneapolis, Minnesota. Association for Computational Linguistics.

Diederik P Kingma and Jimmy Ba. 2014. Adam: A method for stochastic optimization. arXiv preprint arXiv: 1412.6980 .

Philipp Koehn. 2004. Statistical significance tests for machine translation evaluation. In Proceedings of the 2004 Conference on Empirical Methods in Natural Language Processing, pages 388395, Barcelona, Spain. Association for Computational Linguistics.

Terry Koo and Michael Collins. 2010. Efficient thirdorder dependency parsers. In Proceedings of the 48th Annual Meeting of the Association for Computational Linguistics, pages 1-11, Uppsala, Sweden. Association for Computational Linguistics.

Philipp Krähenbühl and Vladlen Koltun. 2011. Efficient inference in fully connected crfs with gaussian edge potentials. In Advances in neural information processing systems, pages 109-117.

Kenton Lee, Luheng He, and Luke Zettlemoyer. 2018. Higher-order coreference resolution with coarse-tofine inference. In Proceedings of the 2018 Conference of the North American Chapter of the Association for Computational Linguistics: Human Language Technologies, Volume 2 (Short Papers), pages 687-692, New Orleans, Louisiana. Association for Computational Linguistics.

Tao Lei, Yuan Zhang, Lluís Màrquez, Alessandro Moschitti, and Regina Barzilay. 2015. High-order lowrank tensors for semantic role labeling. In Proceedings of the 2015 Conference of the North American Chapter of the Association for Computational
Linguistics: Human Language Technologies, pages 1150-1160, Denver, Colorado. Association for Computational Linguistics.

Zuchao Li, Jiaxun Cai, Shexia He, and Hai Zhao. 2018a. Seq2seq dependency parsing. In Proceedings of the 27th International Conference on Computational Linguistics, pages 3203-3214, Santa Fe, New Mexico, USA. Association for Computational Linguistics.

Zuchao Li, Shexia He, Jiaxun Cai, Zhuosheng Zhang, Hai Zhao, Gongshen Liu, Linlin Li, and Luo Si. 2018b. A unified syntax-aware framework for semantic role labeling. In Proceedings of the 2018 Conference on Empirical Methods in Natural Language Processing, pages 2401-2411, Brussels, Belgium. Association for Computational Linguistics.

Zuchao Li, Shexia He, Hai Zhao, Yiqing Zhang, Zhuosheng Zhang, Xi Zhou, and Xiang Zhou. 2019a. Dependency or span, end-to-end uniform semantic role labeling. In Proceedings of the AAAI Conference on Artificial Intelligence, volume 33, pages 6730-6737.

Zuchao Li, Hai Zhao, and Kevin Parnow. 2019b. Global greedy dependency parsing. arXiv preprint arXiv:1911.08673.

Zuchao Li, Hai Zhao, and Kevin Parnow. 2020. Global greedy dependency parsing. In Proceedings of the AAAI Conference on Artificial Intelligence.

Zuchao Li, Hai Zhao, Zhuosheng Zhang, Rui Wang, Masao Utiyama, and Eiichiro Sumita. 2019c. SJTUNICT at MRP 2019: Multi-task learning for end-toend uniform semantic graph parsing. In Proceedings of the Shared Task on Cross-Framework Meaning Representation Parsing at the 2019 Conference on Natural Language Learning, pages 45-54, Hong Kong. Association for Computational Linguistics.

Ying Luo, Fengshun Xiao, and Hai Zhao. 2020. Hierarchical contextualized representation for named entity recognition. In $A A A I$, pages 8441-8448.

Chunchuan Lyu, Shay B. Cohen, and Ivan Titov. 2019. Semantic role labeling with iterative structure refinement. In Proceedings of the 2019 Conference on Empirical Methods in Natural Language Processing and the 9th International Joint Conference on Natural Language Processing (EMNLP-IJCNLP), pages 1071-1082, Hong Kong, China. Association for Computational Linguistics.

Xuezhe Ma and Hai Zhao. 2012. Fourth-order dependency parsing. In Proceedings of COLING 2012: Posters, pages 785-796, Mumbai, India. The COLING 2012 Organizing Committee.

Diego Marcheggiani, Anton Frolov, and Ivan Titov. 2017. A simple and accurate syntax-agnostic neural model for dependency-based semantic role labeling. In Proceedings of the 21st Conference on Computational Natural Language Learning (CoNLL 2017), 
pages 411-420, Vancouver, Canada. Association for Computational Linguistics.

André Martins, Noah Smith, Mário Figueiredo, and Pedro Aguiar. 2011. Dual decomposition with many overlapping components. In Proceedings of the 2011 Conference on Empirical Methods in Natural Language Processing, pages 238-249, Edinburgh, Scotland, UK. Association for Computational Linguistics.

André Martins, Noah Smith, and Eric Xing. 2009. Concise integer linear programming formulations for dependency parsing. In Proceedings of the Joint Conference of the 47th Annual Meeting of the ACL and the 4th International Joint Conference on Natural Language Processing of the AFNLP, pages 342-350, Suntec, Singapore. Association for Computational Linguistics.

André F. T. Martins and Mariana S. C. Almeida. 2014. Priberam: A turbo semantic parser with second order features. In Proceedings of the 8th International Workshop on Semantic Evaluation (SemEval 2014), pages 471-476, Dublin, Ireland. Association for Computational Linguistics.

Yuichiroh Matsubayashi and Kentaro Inui. 2018. Distance-free modeling of multi-predicate interactions in end-to-end Japanese predicate-argument structure analysis. In Proceedings of the 27th International Conference on Computational Linguistics, pages 94-106, Santa Fe, New Mexico, USA. Association for Computational Linguistics.

Ryan McDonald, Koby Crammer, and Fernando Pereira. 2005. Online large-margin training of dependency parsers. In Proceedings of the 43rd Annual Meeting of the Association for Computational Linguistics (ACL'05), pages 91-98, Ann Arbor, Michigan. Association for Computational Linguistics.

Ryan McDonald and Fernando Pereira. 2006. Online learning of approximate dependency parsing algorithms. In 11th Conference of the European Chapter of the Association for Computational Linguistics, Trento, Italy. Association for Computational Linguistics.

Michael C Mozer. 1995. A focused backpropagation algorithm for temporal. Backpropagation: Theory, architectures, and applications, 137.

Phoebe Mulcaire, Swabha Swayamdipta, and Noah A. Smith. 2018. Polyglot semantic role labeling. In Proceedings of the 56th Annual Meeting of the Association for Computational Linguistics (Volume 2: Short Papers), pages 667-672, Melbourne, Australia. Association for Computational Linguistics.

Advanced Language Information Forum NICT. 2020. NICT BERT. https://alaginrc.nict.go.jp/ nict-bert/index.html.
Hiroki Ouchi, Hiroyuki Shindo, Kevin Duh, and Yuji Matsumoto. 2015. Joint case argument identification for Japanese predicate argument structure analysis. In Proceedings of the 53rd Annual Meeting of the Association for Computational Linguistics and the 7th International Joint Conference on Natural Language Processing (Volume 1: Long Papers), pages 961-970, Beijing, China. Association for Computational Linguistics.

Hiroki Ouchi, Hiroyuki Shindo, and Yuji Matsumoto. 2017. Neural modeling of multi-predicate interactions for Japanese predicate argument structure analysis. In Proceedings of the 55th Annual Meeting of the Association for Computational Linguistics (Volume 1: Long Papers), pages 1591-1600, Vancouver, Canada. Association for Computational Linguistics.

Jeffrey Pennington, Richard Socher, and Christopher Manning. 2014. Glove: Global vectors for word representation. In Proceedings of the 2014 Conference on Empirical Methods in Natural Language Processing (EMNLP), pages 1532-1543, Doha, Qatar. Association for Computational Linguistics.

Matthew Peters, Mark Neumann, Mohit Iyyer, Matt Gardner, Christopher Clark, Kenton Lee, and Luke Zettlemoyer. 2018. Deep contextualized word representations. In Proceedings of the 2018 Conference of the North American Chapter of the Association for Computational Linguistics: Human Language Technologies, Volume 1 (Long Papers), pages 2227-2237, New Orleans, Louisiana. Association for Computational Linguistics.

Sashank J. Reddi, Satyen Kale, and Sanjiv Kumar. 2018. On the convergence of adam and beyond. In International Conference on Learning Representations.

Michael Roth and Mirella Lapata. 2016. Neural semantic role labeling with dependency path embeddings. In Proceedings of the 54th Annual Meeting of the Association for Computational Linguistics (Volume 1: Long Papers), pages 1192-1202, Berlin, Germany. Association for Computational Linguistics.

David E Rumelhart, Geoffrey E Hinton, and Ronald J Williams. 1985. Learning internal representations by error propagation. Technical report, California Univ San Diego La Jolla Inst for Cognitive Science.

Tomohide Shibata, Daisuke Kawahara, and Sadao Kurohashi. 2016. Neural network-based model for Japanese predicate argument structure analysis. In Proceedings of the 54th Annual Meeting of the Association for Computational Linguistics (Volume 1: Long Papers), pages 1235-1244, Berlin, Germany. Association for Computational Linguistics.

David Smith and Jason Eisner. 2008. Dependency parsing by belief propagation. In Proceedings of the 2008 Conference on Empirical Methods in Natural Language Processing, pages 145-156, Honolulu, Hawaii. Association for Computational Linguistics. 
Emma Strubell, Patrick Verga, Daniel Andor, David Weiss, and Andrew McCallum. 2018. Linguistically-informed self-attention for semantic role labeling. In Proceedings of the 2018 Conference on Empirical Methods in Natural Language Processing, pages 5027-5038, Brussels, Belgium. Association for Computational Linguistics.

Swabha Swayamdipta, Miguel Ballesteros, Chris Dyer, and Noah A. Smith. 2016. Greedy, joint syntacticsemantic parsing with stack LSTMs. In Proceedings of The 20th SIGNLL Conference on Computational Natural Language Learning, pages 187-197, Berlin, Germany. Association for Computational Linguistics.

Xinyu Wang, Jingxian Huang, and Kewei Tu. 2019. Second-order semantic dependency parsing with end-to-end neural networks. In Proceedings of the 57th Annual Meeting of the Association for Computational Linguistics, pages 4609-4618, Florence, Italy. Association for Computational Linguistics.

Fengshun Xiao, Jiangtong Li, Hai Zhao, Rui Wang, and Kehai Chen. 2019. Lattice-based transformer encoder for neural machine translation. In Proceedings of the 57th Annual Meeting of the Association for Computational Linguistics, pages 3090-3097, Florence, Italy. Association for Computational Linguistics.

Haitong Yang and Chengqing Zong. 2014. Multipredicate semantic role labeling. In Proceedings of the 2014 Conference on Empirical Methods in Natural Language Processing (EMNLP), pages 363373, Doha, Qatar. Association for Computational Linguistics.

Katsumasa Yoshikawa, Masayuki Asahara, and Yuji Matsumoto. 2011. Jointly extracting Japanese predicate-argument relation with Markov Logic. In Proceedings of 5th International Joint Conference on Natural Language Processing, pages 1125-1133, Chiang Mai, Thailand. Asian Federation of Natural Language Processing.

Hao Zhang and Ryan McDonald. 2012. Generalized higher-order dependency parsing with cube pruning. In Proceedings of the 2012 Joint Conference on Empirical Methods in Natural Language Processing and Computational Natural Language Learning, pages 320-331, Jeju Island, Korea. Association for Computational Linguistics.

Yu Zhang, Zhenghua Li, and Min Zhang. 2020. Efficient second-order treecrf for neural dependency parsing. arXiv preprint arXiv:2005.00975.

Zhuosheng Zhang, Yuwei Wu, Zuchao Li, and Hai Zhao. 2019a. Explicit contextual semantics for text comprehension. In Proceedings of the 33rd Pacific Asia Conference on Language, Information and Computation (PACLIC 33).
Zhuosheng Zhang, Yuwei Wu, Hai Zhao, Zuchao Li, Shuailiang Zhang, Xi Zhou, and Xiang Zhou. 2019b. Semantics-aware bert for language understanding. arXiv preprint arXiv:1909.02209.

Zhuosheng Zhang, Hai Zhao, Kangwei Ling, Jiangtong Li, Zuchao Li, Shexia He, and Guohong Fu. 2019c. Effective subword segmentation for text comprehension. IEEE/ACM Transactions on Audio, Speech, and Language Processing, 27(11):1664-1674.

Hai Zhao, Wenliang Chen, Jun'ichi Kazama, Kiyotaka Uchimoto, and Kentaro Torisawa. 2009a. Multilingual dependency learning: Exploiting rich features for tagging syntactic and semantic dependencies. In Proceedings of the Thirteenth Conference on Computational Natural Language Learning (CoNLL 2009): Shared Task, pages 61-66, Boulder, Colorado. Association for Computational Linguistics.

Hai Zhao, Wenliang Chen, and Chunyu Kit. 2009b. Semantic dependency parsing of NomBank and PropBank: An efficient integrated approach via a largescale feature selection. In Proceedings of the 2009 Conference on Empirical Methods in Natural Language Processing, pages 30-39, Singapore. Association for Computational Linguistics.

Hai Zhao and Chunyu Kit. 2008. Parsing syntactic and semantic dependencies with two single-stage maximum entropy models. In CoNLL 2008: Proceedings of the Twelfth Conference on Computational Natural Language Learning, pages 203-207, Manchester, England. Coling 2008 Organizing Committee.

Hai Zhao, Xiaotian Zhang, and Chunyu Kit. 2013. Integrative semantic dependency parsing via efficient large-scale feature selection. Journal of Artificial Intelligence Research, 46:203-233.

Shuai Zheng, Sadeep Jayasumana, Bernardino RomeraParedes, Vibhav Vineet, Zhizhong Su, Dalong Du, Chang Huang, and Philip HS Torr. 2015. Conditional random fields as recurrent neural networks. In Proceedings of the IEEE international conference on computer vision, pages 1529-1537.

Jie Zhou and Wei Xu. 2015. End-to-end learning of semantic role labeling using recurrent neural networks. In Proceedings of the 53rd Annual Meeting of the Association for Computational Linguistics (Volume 1: Long Papers), pages 1127-1137, Beijing, China. Association for Computational Linguistics.

Junru Zhou, Zuchao Li, and Hai Zhao. 2019. Parsing all: Syntax and semantics, dependencies and spans. arXiv preprint arXiv:1908.11522.

Junru Zhou and Hai Zhao. 2019. Head-Driven Phrase Structure Grammar parsing on Penn Treebank. In Proceedings of the 57th Annual Meeting of the Association for Computational Linguistics, pages 2396-2408, Florence, Italy. Association for Computational Linguistics. 


\section{A Appendices}

\section{A.1 Hyper-parameters and Training Details}

In our experiments, the BiLSTM+MLP predicate tagging model only takes words and lemmas as input, and its encoder structure is the same as our main model, so the hyper-parameters are also consistent with our main model. With the BERT+MLP predicate tagging model, the motivation for choosing this instead of using BERT as embedding in the BiLSTM+MLP architecture is to achieve fair comparability with the results of (Zhou et al., 2019).

For the hyper-parameters of our main model, we borrowed most parameter settings from (Dozat and Manning, 2017; Wang et al., 2019), including dropout and initialization strategies. Hyperparameters for our baseline and proposed highorder model are shown in Table 5. We use 100dimensional Glove (Pennington et al., 2014) pretrained word embeddings for English and 300dimensional FastText embeddings (Bojanowski et al., 2017; Grave et al., 2018) for all other languages. As for the pre-training, ELMo(Peters et al., 2018) is only used in English, we take the weighted sum of the 3 layers as the final features, while different versions of BERT(Devlin et al., 2019) are used in different languages, as shown in Table 6, we always use the second-to-last layer outputs as the pre-trained features.

Following the work of (Wang et al., 2019), during model training, the training strategy includes two phases. In the first phase, we used Adam (Kingma and $\mathrm{Ba}, 2014$ ) and annealed the learning rate 0.5 every 10,000 steps. When the training reaches 5,000 steps without improvement, the model optimization enters the second phase; the Adam optimizer is replaced by AMSGrad (Reddi et al., 2018). We trained the model for maximum $100 \mathrm{~K}$ update steps with batch sizes of $\{4 \mathrm{~K}, 2 \mathrm{~K}$, $3 \mathrm{~K}, 4 \mathrm{~K}, 6 \mathrm{~K}, 6 \mathrm{~K}, 6 \mathrm{~K}\}$ tokens for CA, CS, DE, EN, $\mathrm{ES}, \mathrm{JA}$, and $\mathrm{ZH}$, respectively. The training is terminated with an early stopping mechanism when there is no improvement after 10,000 steps on the development sets.

\section{A.2 Detail Results}

The proverb that there is no such thing as a free lunch tells us that no method works in every condition and scope. We explore our proposed high order structure learning for SRL in different languages and conditions: using pre-training or not, given or

\begin{tabular}{lr}
\hline Hidden Layer & Hidden Sizes \\
\hline Word Embedding & 100 (en)/300 (others) \\
Lemma Embedding & 100 \\
Predicate Indicator / Sense Emb & $50 / 50$ \\
ELMo/BERT Linear & 100 \\
Stacked BiLSTM & $3 \times 00$ \\
Biaffine Arc/Label Scorer & 600 \\
Triaffine Arc Scorer & 150 \\
\hline Dropouts & Dropout Prob. \\
\hline Word/Lemma/Predicate & $20 \%$ \\
BiLSTM (FF/recur) & $45 \% / 25 \%$ \\
Biaffine Arc/Label Scorer & $25 \% / 33 \%$ \\
Triaffine Arc Scorer & $25 \%$ \\
\hline Optimizer \& Loss & Value \\
\hline Balance param $\lambda$ & 0.1 \\
Adam $\beta_{1}$ & 0 \\
Adam $\beta_{2}$ & 0.95 \\
Learning rate & $1 e^{-2}$ \\
LR decay & 0.5 \\
L2 regularization & $3 e^{-9}$ \\
\hline
\end{tabular}

Table 5: Hyper-parameters for baseline and high-order SRL models in our experiment.

\begin{tabular}{lcr}
\hline \hline & Version & Provider \\
\hline CA & multi_cased $L-12 \_H-768 \_A-12$ & (Devlin et al., 2019) \\
CS & Slavic BERT: slavic_cased_L-12_H-768_A-12 & (Arkhipov et al., 2019) \\
DE & multi_cased_L-12_H-768_A-12 & (Devlin et al., 2019) \\
EN & wwm_uncased_L-24_H-1024_A-16 & (Devlin et al., 2019) \\
ES & BETO: spanish_wwm_cased_L-12_H-768_A-12 & (Cañete et al., 2020) \\
JA & NICT BERT: japanese_100k_L-12_H-768_A-12 & (NICT, 2020) \\
ZH & chinese_L-12_H-768_A-12 & (Devlin et al., 2019) \\
\hline
\end{tabular}

Table 6: BERT versions for different languages.

not given predicates, and different high-order structure combinations. We report all possible results on development sets, in-domain test sets, and outof-domain test sets in Tables 7, 8, 9 and 10. The experimental results illustrate the following points:

1. In different languages, combinations of high-order structures bring different improvements. Some high-order structure combinations are even worse for performance in some languages.

2. Pre-training can bring about a significant improvement in performance on both in-domain and out-of-domain test sets; however, the in-domain improvement is significantly greater than that of out-of-domain when the two domains are far apart. In particular, the difference between in-domain and out-of-domain in German and English is large, while the two domains in Czech are similar.

3. The SRL results in German are lower than in other languages, the data analysis found that the proportion of predicates is very small, resulting in the sparse targets, which can not train the model well, especially when no predicates are preidentified. 


\begin{tabular}{|c|c|c|c|c|c|c|c|c|c|c|}
\hline \multirow{2}{*}{ Language } & \multirow{2}{*}{ Method } & \multicolumn{3}{|c|}{ Dev } & \multicolumn{3}{|c|}{ Test } & \multicolumn{3}{|c|}{ OOD } \\
\hline & & $\mathrm{P}$ & $\mathrm{R}$ & $\mathrm{F}_{1}$ & $\mathrm{P}$ & $\mathrm{R}$ & $\mathrm{F}_{1}$ & $\mathrm{P}$ & $\mathrm{R}$ & $\mathrm{F}_{1}$ \\
\hline \multirow{16}{*}{$\mathrm{CA}$} & baseline & 85.62 & 83.66 & 84.63 & 85.85 & 84.09 & 84.96 & & & \\
\hline & + sib & 85.91 & 83.98 & 84.94 & 86.09 & 84.46 & 85.27 & & & \\
\hline & + cop & 85.78 & 83.83 & 84.79 & 85.97 & 84.17 & 85.06 & & & \\
\hline & $+g p$ & 86.24 & 83.25 & 84.72 & 86.11 & 83.51 & 84.79 & & & \\
\hline & + sib + cop & 85.70 & 84.25 & 84.97 & 85.90 & 84.85 & 85.37 & & & \\
\hline & $+s i b+g p$ & 85.71 & 84.00 & 84.85 & 85.81 & 84.19 & 84.99 & & & \\
\hline & $+c o p+g p$ & 86.05 & 83.98 & 85.00 & 85.74 & 83.72 & 84.72 & & & \\
\hline & $+\mathbf{A} \mathbf{L} \mathbf{L}$ & 85.64 & 84.09 & 84.86 & 85.67 & 84.24 & 84.95 & & & \\
\hline & $\overline{\text { baseline }^{+B}}$ & 86.95 & 85.74 & 86.34 & 87.10 & 85.72 & 86.40 & & & \\
\hline & $+\mathrm{sib}$ & 86.94 & 85.92 & 86.43 & 87.15 & 86.05 & 86.59 & & & \\
\hline & + cop & 87.15 & 85.96 & 86.55 & 87.14 & 86.00 & 86.57 & & & \\
\hline & $+\mathrm{gp}$ & 87.17 & 86.00 & 86.58 & 86.87 & 85.93 & 86.40 & & & \\
\hline & + sib+cop & 86.96 & 86.57 & 86.76 & 86.89 & 86.53 & 86.71 & & & \\
\hline & $+\mathrm{sib}+\mathrm{gp}$ & 87.26 & 86.55 & 86.90 & 87.10 & 86.40 & 86.75 & & & \\
\hline & $+c o p+g p$ & 87.24 & 86.12 & 86.68 & 87.08 & 85.97 & 86.52 & & & \\
\hline & $+\mathbf{A L L}$ & 87.49 & 86.11 & 86.79 & 87.52 & 86.29 & 86.90 & & & \\
\hline \multirow{16}{*}{ CS } & baseline & 91.20 & 89.79 & 90.49 & 90.87 & 89.49 & 90.18 & 91.22 & 89.88 & 90.54 \\
\hline & $+\mathrm{sib}$ & 91.30 & 89.71 & 90.50 & 91.08 & 89.56 & 90.32 & 91.25 & 89.72 & 90.48 \\
\hline & + cop & 91.40 & 89.77 & 90.58 & 91.09 & 89.56 & 90.32 & 91.27 & 89.75 & 90.51 \\
\hline & $+g p$ & 91.35 & 89.63 & 90.48 & 91.16 & 89.40 & 90.27 & 91.31 & 89.62 & 90.46 \\
\hline & + sib + cop & 91.36 & 89.88 & 90.81 & 91.33 & 89.89 & 90.60 & 91.50 & 90.03 & 90.75 \\
\hline & $+\mathrm{sib}+\mathrm{gp}$ & 91.26 & 89.68 & 90.47 & 91.09 & 89.48 & 90.28 & 91.29 & 89.79 & 90.53 \\
\hline & $+c o p+g p$ & 91.24 & 89.61 & 90.42 & 91.01 & 89.51 & 90.26 & 91.23 & 89.73 & 90.48 \\
\hline & $+\mathbf{A L} \mathbf{L}$ & 91.18 & 89.81 & 90.49 & 90.96 & 89.65 & 90.30 & 91.15 & 89.85 & 90.49 \\
\hline & $\overline{\text { baseline }^{+B}}$ & 92.12 & 91.09 & 91.61 & 91.98 & 90.99 & 91.48 & 91.98 & 91.23 & 91.60 \\
\hline & $+\mathrm{sib}$ & 92.31 & 91.62 & 91.96 & 91.98 & 91.23 & 91.60 & 91.94 & 91.50 & 91.72 \\
\hline & + cop & 92.11 & 91.27 & 91.69 & 92.08 & 91.25 & 91.66 & 91.97 & 91.49 & 91.73 \\
\hline & $+g p$ & 92.02 & 91.19 & 91.60 & 91.97 & 91.20 & 91.58 & 91.85 & 91.32 & 91.59 \\
\hline & $+\mathrm{sib}+\mathrm{cop}$ & 92.04 & 91.30 & 91.67 & 92.38 & 91.49 & 91.93 & 91.84 & 91.47 & 91.65 \\
\hline & $+\mathrm{sib}+\mathrm{gp}$ & 92.11 & 91.24 & 91.68 & 92.06 & 91.15 & 91.61 & 91.84 & 91.41 & 91.63 \\
\hline & $+c o p+g p$ & 91.99 & 91.32 & 91.65 & 91.94 & 91.25 & 91.60 & 91.87 & 91.61 & 91.74 \\
\hline & $+\mathbf{A L L}$ & 92.03 & 91.26 & 91.65 & 91.99 & 91.14 & 91.56 & 91.75 & 91.32 & 91.53 \\
\hline \multirow{16}{*}{$\mathrm{DE}$} & baseline & 75.83 & 72.51 & 74.13 & 77.48 & 74.61 & 76.02 & 71.34 & 67.73 & 69.49 \\
\hline & $+\mathrm{sib}$ & 76.63 & 73.36 & 74.96 & 77.01 & 75.54 & 76.27 & 71.66 & 69.36 & 70.49 \\
\hline & + cop & 74.43 & 72.05 & 73.22 & 76.73 & 74.98 & 75.85 & 69.84 & 68.55 & 69.19 \\
\hline & $+g p$ & 75.69 & 73.02 & 74.33 & 76.33 & 75.11 & 75.71 & 69.74 & 67.35 & 68.53 \\
\hline & $+\mathrm{sib}+\mathrm{cop}$ & 76.24 & 73.25 & 74.72 & 77.53 & 75.33 & 76.41 & 71.28 & 68.88 & 70.06 \\
\hline & $+\mathrm{sib}+\mathrm{gp}$ & 75.29 & 73.02 & 74.14 & 75.86 & 74.74 & 75.29 & 70.15 & 67.79 & 68.95 \\
\hline & $+c o p+g p$ & 76.22 & 72.05 & 74.08 & 77.00 & 74.25 & 75.60 & 69.99 & 66.76 & 68.33 \\
\hline & $+\mathbf{A L L}$ & 75.13 & 72.57 & 73.83 & 76.79 & 74.18 & 75.46 & 71.46 & 67.46 & 69.40 \\
\hline & $\overline{\text { baseline }^{+B}}$ & 84.48 & 82.70 & 83.58 & 85.77 & 84.66 & 85.21 & 71.77 & 70.02 & 70.88 \\
\hline & $+\mathrm{sib}$ & 83.87 & 83.15 & 83.51 & 84.97 & 85.34 & 85.15 & 72.14 & 71.86 & 72.00 \\
\hline & + cop & 84.58 & 83.04 & 83.80 & 84.93 & 85.09 & 85.01 & 71.48 & 71.59 & 71.53 \\
\hline & $+g p$ & 83.82 & 83.15 & 83.49 & 85.01 & 84.53 & 84.77 & 71.80 & 70.67 & 71.23 \\
\hline & + sib + cop & 84.67 & 83.61 & 84.14 & 85.82 & 85.27 & 85.54 & 72.23 & 71.48 & 71.85 \\
\hline & $+\mathrm{sib}+\mathrm{gp}$ & 84.46 & 82.58 & 83.51 & 85.21 & 84.10 & 84.65 & 71.93 & 69.04 & 70.45 \\
\hline & $+c o p+g p$ & 83.49 & 82.30 & 82.89 & 85.20 & 84.41 & 84.80 & 70.88 & 69.69 & 70.28 \\
\hline & $+\mathbf{A L L}$ & 84.53 & 82.70 & 83.60 & 84.95 & 84.17 & 84.56 & 71.39 & 68.17 & 69.74 \\
\hline \multirow{16}{*}{$\mathrm{EN}$} & baseline & 90.15 & 86.27 & 88.17 & 91.29 & 88.00 & 89.61 & 81.37 & 77.12 & 79.19 \\
\hline & $+\mathrm{sib}$ & 89.94 & 86.67 & 88.27 & 91.46 & 88.53 & 89.97 & 81.76 & 77.85 & 79.76 \\
\hline & + cop & 90.03 & 86.59 & 88.27 & 91.40 & 88.45 & 89.90 & 81.89 & 78.07 & 79.94 \\
\hline & $+g p$ & 89.86 & 86.13 & 87.96 & 91.41 & 88.14 & 89.75 & 82.20 & 78.51 & 80.31 \\
\hline & + sib + cop & 90.20 & 86.97 & 88.55 & 91.33 & 88.74 & 90.02 & 81.80 & 78.36 & 80.04 \\
\hline & $+\mathrm{sib}+\mathrm{gp}$ & 90.00 & 86.89 & 88.42 & 91.21 & 88.58 & 89.87 & 81.38 & 77.90 & 79.60 \\
\hline & $+c o p+g p$ & 89.69 & 86.58 & 88.11 & 91.26 & 88.68 & 89.95 & 81.32 & 78.02 & 79.64 \\
\hline & $+\mathbf{A L L}$ & 90.03 & 86.91 & 88.44 & 91.60 & 88.95 & 90.26 & 82.6 & 78.75 & 80.63 \\
\hline & baseline $^{+\mathrm{B}}$ & 91.35 & 88.84 & 90.08 & 92.31 & 90.18 & 91.23 & 86.14 & 83.49 & 84.79 \\
\hline & $+\mathrm{sib}$ & 91.3 & 89.11 & 90.19 & 92.49 & 90.58 & 91.53 & 85.96 & 83.97 & 84.95 \\
\hline & + cop & 91.3 & 89.19 & 90.23 & 92.21 & 90.5 & 91.35 & 86.03 & 84.07 & 85.04 \\
\hline & $+\mathrm{gp}$ & 91.66 & 88.6 & 90.11 & 92.77 & 90.09 & 91.41 & 86.22 & 83.1 & 84.63 \\
\hline & + sib + cop & 91.16 & 89.6 & 90.37 & 92.36 & 90.84 & 91.6 & 85.59 & 83.92 & 84.75 \\
\hline & $+\mathrm{sib}+\mathrm{gp}$ & 91.62 & 88.99 & 90.28 & 92.64 & 90.44 & 91.53 & 86.33 & 83.61 & 84.95 \\
\hline & $+c o p+g p$ & 91.27 & 89.02 & 90.14 & 92.37 & 90.35 & 91.35 & 86.03 & 83.88 & 84.94 \\
\hline & $+\mathbf{A L L}$ & 91.56 & 89.35 & 90.44 & 92.59 & 90.98 & 91.77 & 86.49 & 83.80 & 85.13 \\
\hline
\end{tabular}

Table 7: w/ pre-identified predicate results. 


\begin{tabular}{|c|c|c|c|c|c|c|c|c|c|c|}
\hline \multirow{2}{*}{ Language } & \multirow{2}{*}{ Method } & \multicolumn{3}{|c|}{ Dev } & \multicolumn{3}{|c|}{ Test } & \multicolumn{3}{|c|}{ OOD } \\
\hline & & $\mathrm{P}$ & $\mathrm{R}$ & $\mathrm{F}_{1}$ & $\mathrm{P}$ & $\mathrm{R}$ & $\mathrm{F}_{1}$ & $\mathrm{P}$ & $\mathrm{R}$ & $\overline{F_{1}}$ \\
\hline \multirow{16}{*}{ ES } & baseline & 84.58 & 82.58 & 83.57 & 84.97 & 82.60 & 83.77 & & & \\
\hline & $+\mathrm{sib}$ & 84.90 & 83.11 & 83.99 & 85.18 & 83.28 & 84.22 & & & \\
\hline & + cop & 84.66 & 82.76 & 83.70 & 85.12 & 83.21 & 84.15 & & & \\
\hline & $+g p$ & 84.95 & 82.33 & 83.62 & 85.51 & 82.31 & 83.88 & & & \\
\hline & $+\mathrm{sib}+\mathrm{cop}$ & 84.66 & 82.98 & 83.81 & 85.36 & 83.45 & 84.39 & & & \\
\hline & $+\mathrm{sib}+\mathrm{gp}$ & 84.73 & 83.13 & 83.92 & 85.05 & 83.21 & 84.12 & & & \\
\hline & $+c o p+g p$ & 84.80 & 82.42 & 83.59 & 85.35 & 82.87 & 84.09 & & & \\
\hline & $+\mathbf{A L L}$ & 84.89 & 82.90 & 83.89 & 85.12 & 83.29 & 84.20 & & & \\
\hline & baseline $^{+\mathrm{B}}$ & 87.14 & 85.91 & 86.52 & 87.23 & 85.98 & 86.60 & & & \\
\hline & $+\mathrm{sib}$ & 87.36 & 85.62 & 86.48 & 87.48 & 85.97 & 86.72 & & & \\
\hline & + cop & 87.03 & 85.94 & 86.48 & 87.19 & 86.11 & 86.65 & & & \\
\hline & $+g p$ & 87.21 & 86.04 & 86.62 & 87.22 & 85.95 & 86.58 & & & \\
\hline & $+\mathrm{sib}+\mathrm{cop}$ & 86.98 & 86.45 & 86.71 & 87.24 & 86.67 & 86.96 & & & \\
\hline & $+\mathrm{sib}+\mathrm{gp}$ & 87.62 & 85.66 & 86.63 & 87.62 & 85.84 & 86.72 & & & \\
\hline & $+c o p+g p$ & 87.26 & 85.85 & 86.55 & 87.09 & 85.82 & 86.45 & & & \\
\hline & $+\mathbf{A L L}$ & 87.49 & 86.11 & 86.79 & 87.52 & 86.29 & 86.90 & & & \\
\hline \multirow{16}{*}{ JA } & baseline & 88.49 & 76.68 & 82.16 & 88.15 & 77.79 & 82.65 & & & \\
\hline & $+\mathrm{sib}$ & 87.30 & 78.45 & 82.64 & 86.14 & 79.85 & 82.88 & & & \\
\hline & $+\mathrm{cop}$ & 87.71 & 77.22 & 82.13 & 87.90 & 78.58 & 82.98 & & & \\
\hline & $+g p$ & 86.65 & 77.15 & 81.63 & 86.03 & 78.62 & 82.16 & & & \\
\hline & $+\mathrm{sib}+\mathrm{cop}$ & 87.97 & 78.78 & 83.12 & 87.51 & 79.38 & 83.25 & & & \\
\hline & $+\mathrm{sib}+\mathrm{gp}$ & 88.32 & 77.72 & 82.68 & 88.34 & 78.51 & 83.14 & & & \\
\hline & $+\mathrm{cop}+\mathrm{gp}$ & 88.36 & 76.99 & 82.28 & 88.09 & 78.04 & 82.76 & & & \\
\hline & $+\mathbf{A L L}$ & 88.86 & 77.18 & 82.61 & 88.17 & 78.28 & 82.93 & & & \\
\hline & $\overline{\text { baseline }^{+\mathrm{B}}}$ & 89.93 & 80.89 & 85.17 & 89.63 & 81.83 & 85.55 & & & \\
\hline & $+\mathrm{sib}$ & 89.29 & 81.06 & 84.98 & 89.02 & 82.08 & 85.41 & & & \\
\hline & + cop & 89.99 & 80.56 & 85.02 & 89.71 & 81.57 & 85.45 & & & \\
\hline & $+g p$ & 89.43 & 80.40 & 84.67 & 88.88 & 81.08 & 84.80 & & & \\
\hline & $+\mathrm{sib}+\mathrm{cop}$ & 88.67 & 82.34 & 85.39 & 88.65 & 83.32 & 85.90 & & & \\
\hline & $+\mathrm{sib}+\mathrm{gp}$ & 89.90 & 80.05 & 84.69 & 89.75 & 81.27 & 85.30 & & & \\
\hline & $+c o p+g p$ & 89.20 & 81.27 & 85.05 & 88.59 & 82.03 & 85.19 & & & \\
\hline & $+\mathbf{A L L}$ & 90.51 & 80.33 & 85.12 & 89.69 & 81.66 & 85.49 & & & \\
\hline \multirow{16}{*}{$\mathrm{ZH}$} & baseline & 87.28 & 83.84 & 85.52 & 87.95 & 83.63 & 85.73 & & & \\
\hline & + sib & 87.58 & 83.96 & 85.73 & 87.94 & 83.80 & 85.82 & & & \\
\hline & $+\mathrm{cop}$ & 88.33 & 83.40 & 85.80 & 88.61 & 83.29 & 85.87 & & & \\
\hline & $+g p$ & 88.08 & 82.25 & 85.07 & 88.46 & 82.01 & 85.12 & & & \\
\hline & $+\mathrm{sib}+\mathrm{cop}$ & 87.95 & 83.81 & 85.83 & 88.09 & 83.64 & 85.81 & & & \\
\hline & + sib $+g p$ & 88.28 & 83.42 & 85.78 & 88.20 & 83.26 & 85.66 & & & \\
\hline & $+c o p+g p$ & 88.38 & 82.56 & 85.37 & 88.54 & 82.63 & 85.48 & & & \\
\hline & $+\mathbf{A L L}$ & 88.44 & 83.40 & 85.85 & 88.35 & 83.82 & 86.02 & & & \\
\hline & baseline $^{+\mathrm{B}}$ & 89.63 & 86.69 & 88.13 & 89.94 & 86.60 & 88.24 & & & \\
\hline & $+\mathrm{sib}$ & 89.47 & 87.40 & 88.42 & 89.64 & 87.34 & 88.48 & & & \\
\hline & + cop & 89.63 & 87.35 & 88.48 & 89.79 & 87.33 & 88.54 & & & \\
\hline & $+g p$ & 89.16 & 86.74 & 87.93 & 89.54 & 86.69 & 88.09 & & & \\
\hline & $+\mathrm{sib}+\mathrm{cop}$ & 89.80 & 86.92 & 88.34 & 89.97 & 87.45 & 88.69 & & & \\
\hline & $+\mathrm{sib}+\mathrm{gp}$ & 89.60 & 87.39 & 88.48 & 89.79 & 87.30 & 88.53 & & & \\
\hline & $+c o p+g p$ & 89.48 & 87.18 & 88.32 & 89.70 & 87.36 & 88.52 & & & \\
\hline & $+\mathbf{A L L}$ & 88.95 & 87.58 & 88.26 & 89.07 & 87.71 & 88.38 & & & \\
\hline
\end{tabular}

Table 8: w/ pre-identified predicate results. 


\begin{tabular}{|c|c|c|c|c|c|c|c|c|c|c|}
\hline \multirow{2}{*}{ Language } & \multirow{2}{*}{ Method } & \multicolumn{3}{|c|}{ Dev } & \multicolumn{3}{|c|}{ Test } & \multicolumn{3}{|c|}{ OOD } \\
\hline & & $\mathrm{P}$ & $\mathrm{R}$ & $\mathrm{F}_{1}$ & $\mathrm{P}$ & $\mathrm{R}$ & $\mathrm{F}_{1}$ & $\mathrm{P}$ & $\mathrm{R}$ & $\mathrm{F}_{1}$ \\
\hline \multirow{16}{*}{$\mathrm{CA}$} & baseline & 83.97 & 82.62 & 83.29 & 84.45 & 82.93 & 83.69 & & & \\
\hline & + sib & 84.17 & 82.97 & 83.57 & 84.57 & 83.21 & 83.89 & & & \\
\hline & + cop & 84.21 & 82.85 & 83.52 & 84.38 & 82.94 & 83.66 & & & \\
\hline & $+g p$ & 84.52 & 82.23 & 83.36 & 84.55 & 82.28 & 83.40 & & & \\
\hline & + sib + cop & 84.03 & 83.15 & 83.59 & 84.69 & 83.46 & 84.07 & & & \\
\hline & $+s i b+g p$ & 84.15 & 83.06 & 83.60 & 84.35 & 83.07 & 83.70 & & & \\
\hline & $+c o p+g p$ & 84.44 & 82.93 & 83.68 & 84.32 & 82.64 & 83.47 & & & \\
\hline & $+\mathbf{A L L}$ & 83.95 & 83.07 & 83.51 & 84.14 & 83.10 & 83.62 & & & \\
\hline & $\overline{\text { baseline }^{+B}}$ & 85.05 & 84.47 & 84.76 & 85.51 & 84.73 & 85.12 & & & \\
\hline & $+\mathrm{sib}$ & 85.14 & 84.66 & 84.90 & 85.60 & 85.10 & 85.35 & & & \\
\hline & + cop & 85.46 & 84.80 & 85.13 & 85.57 & 84.99 & 85.28 & & & \\
\hline & $+g p$ & 85.33 & 84.81 & 85.07 & 85.29 & 84.93 & 85.11 & & & \\
\hline & + sib+cop & 85.39 & 85.27 & 85.33 & 85.47 & 85.38 & 85.42 & & & \\
\hline & $+\mathrm{sib}+\mathrm{gp}$ & 85.39 & 85.27 & 85.33 & 85.47 & 85.38 & 85.42 & & & \\
\hline & $+c o p+g p$ & 85.39 & 84.89 & 85.14 & 85.53 & 85.05 & 85.29 & & & \\
\hline & $+\mathbf{A L L}$ & 86.08 & 85.35 & 85.72 & 86.15 & 85.49 & 85.82 & & & \\
\hline \multirow{16}{*}{ CS } & baseline & 90.25 & 88.84 & 89.54 & 89.98 & 88.47 & 89.22 & 89.98 & 88.47 & 89.22 \\
\hline & $+\mathrm{sib}$ & 90.37 & 88.78 & 89.57 & 90.17 & 88.52 & 89.34 & 89.89 & 88.44 & 89.16 \\
\hline & + cop & 90.43 & 88.82 & 89.62 & 90.16 & 88.53 & 89.34 & 89.86 & 88.45 & 89.15 \\
\hline & $+g p$ & 90.38 & 88.70 & 89.53 & 90.21 & 88.34 & 89.26 & 89.86 & 88.30 & 89.07 \\
\hline & + sib + cop & 90.73 & 88.94 & 89.82 & 90.21 & 88.65 & 89.42 & 89.85 & 88.49 & 89.16 \\
\hline & $+\mathrm{sib}+\mathrm{gp}$ & 90.34 & 88.77 & 89.55 & 90.15 & 88.41 & 89.27 & 89.88 & 88.51 & 89.19 \\
\hline & $+c o p+g p$ & 90.32 & 88.72 & 89.51 & 90.12 & 88.47 & 89.29 & 89.86 & 88.44 & 89.14 \\
\hline & $+\mathbf{A L} \mathbf{L}$ & 90.28 & 88.90 & 89.58 & 90.25 & 88.68 & 89.45 & 89.82 & 88.85 & 89.33 \\
\hline & $\overline{\text { baseline }^{+B}}$ & 91.32 & 90.42 & 90.87 & 91.25 & 90.20 & 90.72 & 90.97 & 90.30 & 90.63 \\
\hline & $+\mathrm{sib}$ & 91.29 & 90.61 & 90.95 & 91.22 & 90.45 & 90.83 & 90.89 & 90.56 & 90.72 \\
\hline & + cop & 91.33 & 90.59 & 90.96 & 91.32 & 90.50 & 90.91 & 90.99 & 90.58 & 90.78 \\
\hline & $+g p$ & 91.22 & 90.52 & 90.87 & 91.21 & 90.45 & 90.83 & 90.87 & 90.37 & 90.62 \\
\hline & + sib + cop & 91.25 & 90.63 & 90.94 & 91.23 & 91.21 & 91.22 & 90.84 & 90.55 & 90.69 \\
\hline & $+\mathrm{sib}+\mathrm{gp}$ & 91.31 & 90.55 & 90.93 & 91.31 & 90.37 & 90.84 & 90.82 & 90.48 & 90.65 \\
\hline & $+c o p+g p$ & 91.18 & 90.63 & 90.91 & 91.20 & 90.47 & 90.83 & 90.84 & 90.66 & 90.75 \\
\hline & $+\mathbf{A L L}$ & 91.22 & 90.55 & 90.89 & 91.23 & 90.37 & 90.80 & 90.74 & 90.42 & 90.58 \\
\hline \multirow{16}{*}{$\mathrm{DE}$} & baseline & 53.59 & 68.87 & 60.27 & 51.03 & 72.95 & 60.06 & 39.97 & 45.14 & 42.40 \\
\hline & $+\mathrm{sib}$ & 53.81 & 69.89 & 60.81 & 51.32 & 73.63 & 60.48 & 40.26 & 46.01 & 42.94 \\
\hline & + cop & 52.34 & 68.70 & 59.41 & 50.41 & 72.70 & 59.54 & 39.82 & 45.79 & 42.60 \\
\hline & $+g p$ & 53.42 & 69.72 & 60.49 & 50.72 & 73.38 & 59.98 & 40.19 & 45.30 & 42.59 \\
\hline & $+\mathrm{sib}+\mathrm{cop}$ & 53.28 & 70.29 & 60.61 & 50.79 & 73.44 & 60.05 & 40.67 & 45.95 & 43.15 \\
\hline & $+\mathrm{sib}+\mathrm{gp}$ & 53.15 & 69.72 & 60.32 & 50.21 & 72.58 & 59.36 & 39.92 & 45.19 & 42.39 \\
\hline & $+c o p+g p$ & 53.13 & 68.53 & 59.86 & 51.17 & 72.77 & 60.09 & 40.14 & 44.87 & 42.37 \\
\hline & $+\mathbf{A L L}$ & 53.10 & 69.21 & 60.09 & 50.67 & 72.46 & 59.63 & 40.59 & 44.98 & 42.67 \\
\hline & $\overline{\text { baseline }^{+B}}$ & 57.87 & 80.14 & 67.21 & 55.67 & 83.18 & 66.70 & 37.99 & 43.13 & 40.40 \\
\hline & $+\mathrm{sib}$ & 57.68 & 80.76 & 67.30 & 55.70 & 83.98 & 66.98 & 39.00 & 44.38 & 41.51 \\
\hline & + cop & 57.71 & 80.71 & 67.30 & 55.66 & 83.61 & 66.83 & 38.00 & 44.05 & 40.81 \\
\hline & $+g p$ & 57.52 & 80.54 & 67.11 & 55.35 & 83.12 & 66.45 & 38.53 & 43.89 & 41.04 \\
\hline & + sib + cop & 58.00 & 81.33 & 67.71 & 55.86 & 84.17 & 67.15 & 38.94 & 44.38 & 41.48 \\
\hline & $+s i b+g p$ & 58.18 & 80.36 & 67.50 & 55.43 & 82.44 & 66.29 & 38.66 & 43.07 & 40.75 \\
\hline & $+c o p+g p$ & 57.23 & 79.97 & 66.71 & 55.43 & 82.99 & 66.47 & 38.34 & 42.86 & 40.47 \\
\hline & $+\mathbf{A L L}$ & 58.38 & 80.48 & 67.67 & 55.57 & 82.62 & 66.45 & 37.67 & 42.15 & 39.78 \\
\hline \multirow{16}{*}{$\mathrm{EN}$} & baseline & 85.18 & 82.58 & 83.86 & 86.12 & 85.34 & 85.73 & 74.51 & 73.48 & 73.99 \\
\hline & $+\mathrm{sib}$ & 85.36 & 83.21 & 84.27 & 86.00 & 85.64 & 85.82 & 74.38 & 73.31 & 73.84 \\
\hline & + cop & 85.31 & 82.91 & 84.09 & 86.12 & 85.56 & 85.84 & 74.35 & 73.07 & 73.70 \\
\hline & $+g p$ & 85.40 & 82.67 & 84.01 & 86.04 & 85.13 & 85.58 & 74.43 & 72.68 & 73.55 \\
\hline & + sib + cop & 85.15 & 83.17 & 84.15 & 86.26 & 86.06 & 86.16 & 74.76 & 73.65 & 74.20 \\
\hline & $+\mathrm{sib}+\mathrm{gp}$ & 85.16 & 83.22 & 84.18 & 85.82 & 85.59 & 85.71 & 74.18 & 72.92 & 73.55 \\
\hline & $+c o p+g p$ & 84.93 & 83.07 & 83.99 & 86.00 & 85.59 & 85.79 & 74.29 & 73.31 & 73.80 \\
\hline & $+\mathbf{A L L}$ & 85.10 & 83.00 & 84.04 & 86.16 & 85.56 & 85.86 & 74.65 & 73.17 & 73.90 \\
\hline & baseline $^{+\mathrm{B}}$ & 88.21 & 85.64 & 86.90 & 88.51 & 88.05 & 88.28 & 80.49 & 79.65 & 80.07 \\
\hline & $+\mathrm{sib}$ & 88.19 & 85.88 & 87.02 & 88.66 & 88.39 & 88.52 & 80.41 & 79.84 & 80.13 \\
\hline & + cop & 88.13 & 86.00 & 87.05 & 88.39 & 88.30 & 88.34 & 80.32 & 80.26 & 80.29 \\
\hline & $+\mathrm{gp}$ & 88.54 & 85.41 & 86.95 & 89.01 & 87.98 & 88.49 & 80.63 & 79.24 & 79.93 \\
\hline & + sib + cop & 88.01 & 86.40 & 87.20 & 88.55 & 88.60 & 88.57 & 79.87 & 79.89 & 79.88 \\
\hline & $+\mathrm{sib}+\mathrm{gp}$ & 88.38 & 85.78 & 87.06 & 88.82 & 88.19 & 88.50 & 80.57 & 79.55 & 80.06 \\
\hline & $+c o p+g p$ & 88.16 & 85.79 & 86.96 & 88.59 & 88.20 & 88.40 & 80.35 & 80.11 & 80.23 \\
\hline & $+\mathbf{A L L}$ & 87.98 & 86.25 & 87.11 & 88.77 & 88.62 & 88.70 & 80.01 & 79.80 & 79.90 \\
\hline
\end{tabular}

Table 9: w/o pre-identified predicate results. 


\begin{tabular}{|c|c|c|c|c|c|c|c|c|c|c|}
\hline \multirow{2}{*}{ Language } & \multirow{2}{*}{ Method } & \multicolumn{3}{|c|}{ Dev } & \multicolumn{3}{|c|}{ Test } & \multicolumn{3}{|c|}{ OOD } \\
\hline & & $P$ & $\mathrm{R}$ & $\mathrm{F}_{1}$ & $\mathrm{P}$ & $\mathrm{R}$ & $\mathrm{F}_{1}$ & $\mathrm{P}$ & $\mathrm{R}$ & $\mathrm{F}_{1}$ \\
\hline \multirow{16}{*}{ ES } & baseline & 83.35 & 81.79 & 82.57 & 83.52 & 81.58 & 82.54 & & & \\
\hline & $+\mathrm{sib}$ & 83.72 & 82.42 & 83.06 & 83.66 & 82.26 & 82.96 & & & \\
\hline & + cop & 83.59 & 82.09 & 82.84 & 83.70 & 82.15 & 82.91 & & & \\
\hline & $+g p$ & 83.74 & 81.57 & 82.64 & 83.98 & 81.18 & 82.55 & & & \\
\hline & $+\mathrm{sib}+\mathrm{cop}$ & 83.41 & 82.11 & 82.75 & 83.87 & 82.38 & 83.11 & & & \\
\hline & $+\mathrm{sib}+\mathrm{gp}$ & 83.56 & 82.35 & 82.95 & 83.49 & 82.18 & 82.83 & & & \\
\hline & $+\mathrm{cop}+\mathrm{gp}$ & 83.65 & 81.61 & 82.62 & 83.87 & 81.86 & 82.85 & & & \\
\hline & $+\mathbf{A L L}$ & 83.72 & 82.09 & 82.90 & 83.64 & 82.18 & 82.90 & & & \\
\hline & baseline $^{+\mathrm{B}}$ & 85.88 & 85.21 & 85.55 & 85.92 & 85.09 & 85.50 & & & \\
\hline & $+\mathrm{sib}$ & 85.99 & 84.89 & 85.44 & 86.19 & 85.13 & 85.66 & & & \\
\hline & + cop & 85.81 & 85.28 & 85.55 & 85.90 & 85.27 & 85.58 & & & \\
\hline & $+g p$ & 85.85 & 85.30 & 85.57 & 85.84 & 85.12 & 85.48 & & & \\
\hline & + sib+cop & 85.66 & 85.64 & 85.65 & 86.21 & 85.75 & 86.00 & & & \\
\hline & + sib+gp & 86.20 & 84.93 & 85.56 & 86.32 & 85.02 & 85.66 & & & \\
\hline & $+c o p+g p$ & 85.88 & 85.09 & 85.48 & 85.76 & 84.94 & 85.35 & & & \\
\hline & $+\mathbf{A L L}$ & 86.08 & 85.35 & 85.72 & 86.15 & 85.49 & 85.82 & & & \\
\hline \multirow{16}{*}{ JA } & baseline & 79.86 & 68.68 & 73.85 & 79.67 & 68.53 & 73.68 & & & \\
\hline & $+\mathrm{sib}$ & 78.92 & 70.12 & 74.26 & 77.77 & 70.01 & 73.69 & & & \\
\hline & $+\mathrm{cop}$ & 79.51 & 68.92 & 73.83 & 79.48 & 69.23 & 74.00 & & & \\
\hline & $+g p$ & 79.23 & 69.39 & 73.98 & 78.14 & 69.24 & 73.42 & & & \\
\hline & $+\mathrm{sib}+\mathrm{cop}$ & 79.78 & 70.47 & 74.84 & 79.14 & 69.57 & 74.05 & & & \\
\hline & + sib+gp & 79.99 & 69.22 & 74.22 & 79.77 & 68.98 & 73.98 & & & \\
\hline & $+\mathrm{cop}+\mathrm{gp}$ & 80.45 & 69.04 & 74.31 & 79.91 & 69.01 & 74.06 & & & \\
\hline & $+\mathbf{A L L}$ & 80.37 & 69.06 & 74.28 & 80.04 & 69.16 & 74.20 & & & \\
\hline & baseline $^{+B}$ & 81.20 & 74.84 & 77.89 & 82.03 & 74.24 & 77.94 & & & \\
\hline & $+\mathrm{sib}$ & 82.77 & 74.49 & 78.41 & 80.53 & 76.86 & 78.65 & & & \\
\hline & + cop & 82.61 & 74.35 & 78.27 & 82.33 & 75.36 & 78.69 & & & \\
\hline & $+g p$ & 82.15 & 74.16 & 77.95 & 80.98 & 75.48 & 78.13 & & & \\
\hline & $+\mathrm{sib}+\mathrm{cop}$ & 81.55 & 75.84 & 78.59 & 82.16 & 75.85 & 78.88 & & & \\
\hline & $+\mathrm{sib}+\mathrm{gp}$ & 82.28 & 73.62 & 77.71 & 82.10 & 75.04 & 78.41 & & & \\
\hline & $+c o p+g p$ & 82.14 & 74.89 & 78.35 & 81.35 & 75.73 & 78.44 & & & \\
\hline & $+\mathbf{A L} \mathbf{L}$ & 82.72 & 74.21 & 78.23 & 81.88 & 75.39 & 78.50 & & & \\
\hline \multirow{16}{*}{$\mathrm{ZH}$} & baseline & 82.00 & 80.20 & 81.09 & 83.08 & 79.91 & 81.46 & & & \\
\hline & $+\mathrm{sib}$ & 82.34 & 80.42 & 81.37 & 83.04 & 80.00 & 81.50 & & & \\
\hline & + cop & 83.04 & 79.81 & 81.40 & 83.65 & 79.50 & 81.52 & & & \\
\hline & $+g p$ & 82.84 & 78.73 & 80.73 & 83.71 & 78.25 & 80.89 & & & \\
\hline & $+\mathrm{sib}+\mathrm{cop}$ & 82.70 & 80.19 & 81.43 & 83.07 & 80.99 & 82.01 & & & \\
\hline & $+\mathrm{sib}+\mathrm{gp}$ & 82.87 & 79.89 & 81.35 & 83.31 & 79.52 & 81.37 & & & \\
\hline & $+c o p+g p$ & 82.97 & 79.07 & 80.98 & 83.63 & 78.87 & 81.18 & & & \\
\hline & $+\mathbf{A L L}$ & 83.19 & 79.86 & 81.49 & 83.51 & 79.59 & 81.50 & & & \\
\hline & baseline $^{+B}$ & 86.42 & 84.20 & 85.29 & 86.82 & 83.98 & 85.38 & & & \\
\hline & + sib & 86.24 & 84.78 & 85.51 & 86.47 & 84.63 & 85.54 & & & \\
\hline & + cop & 86.34 & 84.72 & 85.52 & 86.55 & 84.54 & 85.53 & & & \\
\hline & $+g p$ & 85.90 & 84.24 & 85.07 & 86.37 & 84.00 & 85.17 & & & \\
\hline & + sib+cop & 86.53 & 84.61 & 85.56 & 86.83 & 84.57 & 85.68 & & & \\
\hline & $+\mathrm{sib}+\mathrm{gp}$ & 86.33 & 84.74 & 85.53 & 86.64 & 84.58 & 85.60 & & & \\
\hline & $+c o p+g p$ & 86.28 & 84.63 & 85.45 & 86.61 & 84.66 & 85.63 & & & \\
\hline & $+\mathbf{A L L}$ & 85.63 & 84.99 & 85.31 & 85.90 & 84.90 & 85.39 & & & \\
\hline
\end{tabular}

Table 10: w/o pre-identified predicate results. 